\title{
Tagged-particle dynamics in a fluid adsorbed in a disordered porous solid: interplay between the diffusion-localization and liquid-glass transitions
}

\author{
V. Krakoviack \\ Laboratoire de Chimie, UMR CNRS 5182, École Normale Supérieure de Lyon, 46 Allée d'Italie, 69364 Lyon cedex 07, France
}

(Dated: June 5, 2018)

\begin{abstract}
A mode-coupling theory for the slow single-particle dynamics in fluids adsorbed in disordered porous media is derived, which complements previous work on the collective dynamics [V. Krakoviack, Phys. Rev. E 75, 031503 (2007)]. Its equations, like the previous ones, reflect the interplay between confinement-induced relaxation phenomena and glassy dynamics through the presence of two contributions in the slow part of the memory kernel, which are linear and quadratic in the density correlation functions, respectively. From numerical solutions for two simple models with pure hard core interactions, it is shown that two different scenarios result for the diffusion-localization transition, depending on the strength of the confinement. For weak confinement, this transition is discontinuous and coincides with the ideal glass transition, like in one-component bulk systems, while, for strong confinement, it is continuous and occurs before the collective dynamics gets nonergodic. In the latter case, the glass transition manifests itself as a secondary transition, which can be either continuous or discontinuous, in the already arrested single-particle dynamics. The main features of the anomalous dynamics found in the vicinity of all these transitions are reviewed and illustrated with detailed computations.
\end{abstract}

\section{INTRODUCTION}

The dynamics of fluids confined at the nanoscale is a topic of great interest, both for fundamental and practical reasons $[1,2,3]$. Among the relevant questions from a fundamental point of view, one counts anomalous molecular transport characterized by subdiffusive laws, which result from the trapping and obstruction phenomena due to geometric and topological constraints and/or quenched disorder $[4,5,6]$. Another topic which has attracted considerable attention in the past few years is the dynamics of confined glass-forming liquids [7, 8], with the main motivation of clarifying and substantiating the concept of cooperativity, a key ingredient of many glass transition theories $[9,10,11]$. On the practical side, the study of many problems in the fields of biology, geology, and chemical engineering could benefit from a finer understanding of the dynamical behavior of confined fluids.

Recently, as a first step towards a general and versatile theoretical approach to the problem, we have developed an extension of the ideal mode-coupling theory (MCT) of the liquid-glass transition $[12,13,14,15,16]$ which allows one to study the slow collective dynamics of fluids adsorbed in disordered porous solids on the basis of the model of the so-called "quenched-annealed" (QA) binary mixture $[17,18,19]$. In these systems, first introduced by Madden and Glandt [20, 21], the porous solid (the quenched component) is represented by a random array of particles frozen in a disordered configuration sampled from a given probability distribution, in which the fluid molecules (the annealed component) equilibrate.

The theory makes a number of interesting predictions $[17,18,19]$. Indeed, two distinct types of ideal liquidglass transition scenarios are predicted, which are either discontinuous, like in the bulk, in situations of weak confinement (dilute matrices), or continuous in situations of strong confinement (dense matrices). In the intermediate region where the nature of the transition changes, degenerate or genuine higher-order singularities and glass-glass transition lines are found depending on the details of the fluid-solid system. Moreover, a reentrant glass transition line is predicted in the low fluid-high matrix density regime.

In the present paper, we address within the same theoretical framework the dynamics of a tagged particle moving in a fluid adsorbed in a disordered porous solid. More precisely, we shall derive and solve the MCT equations describing the time evolution of the wave-vectordependent tagged-particle density correlators and, from their small wave vector limit, of the mean-squared displacement. These quantities can be measured by a variety of techniques and usually more easily than the corresponding collective properties. For instance, because hydrogen has a large incoherent scattering length, inelastic neutron scattering techniques essentially probe the spectrum of the incoherent intermediate scattering function when applied to hydrogen-rich molecular systems like water and many organic glassformers; deuterated samples are required to measure its coherent counterpart. Also, in computer simulations, tagged-particle quantities can be averaged over all fluid particles in the sample, leading to a very significant improvement in statistics compared to the collective functions. Thus, we anticipate that with the present developments it will become easier to compare the predictions of the theory with high quality experimental and simulation data.

A priori, one expects to see manifestations of two types of ergodicity breaking events in the present problem. The ideal liquid-glass transition as obtained from the study of the collective dynamics is the first one, following the same mechanism as in the bulk: the tagged-particle dynamics is coupled to the collective dynamics and a spontaneous arrest of the latter might trigger a similar arrest of the former. The second one is the diffusion-localization tran- 
sition corresponding to the permanent trapping of the tagged particle in some domain of finite spatial extent. This is a confinement- and disorder-induced phenomenon which is already present when the tagged particle is alone in the porous medium. It is one aim of the present work to understand how these two processes interplay. Note that early MCT studies of bulk binary hard sphere mixtures have clearly shown that the relation between the two phenomena can be nontrivial [22, 23, 24].

From the point of view of the diffusion-localization transition, the reported theory represents an extension of early work on the classical random Lorentz gas $[25,26,27]$, incorporating collective effects due to finite fluid densities at two levels. First, through the use of the proper statistical mechanical formalism for the definition and the computation of the static correlation functions of the QA mixture which are needed as input of the dynamical theory, the dependence of the structure of the fluid on its density and on the fluid-fluid interactions is naturally accounted for. Second, as already mentioned, the coupling of the tagged-particle dynamics to the collective dynamics is explicitly included. So, in addition to the localization effect of the porous medium, obstruction phenomena due to molecular crowding or, conversely, decorrelation processes due to fluid-fluid collisions can in principle be captured by the theory.

The paper is organized as follows. In Sec. II, the model of the QA mixture is defined, the MCT equations for the collective dynamics are recalled and those for the taggedparticle dynamics are derived and shortly discussed. In Sec. III, the relation of the theory to previous work and a variant are explored, in an attempt to provide guidelines for future studies with other formalisms. The dynamical phase diagrams are considered in more details in Sec. IV. Section V is devoted to the presentation of the complete relaxation scenarios and Section VI to concluding remarks. A few technical results are presented in appendices.

\section{THE MODEL AND ITS MODE-COUPLING THEORY}

As in MCT studies of bulk systems, the variables of interest in the present theory are the Fourier components of the microscopic densities characterizing the system. For the problem of the dynamics of a tagged particle moving in a fluid adsorbed in a disordered porous solid (see Fig. 1 for a sketch of the system), there are four such quantities which are relevant either to the statics or the dynamics.

In a QA system, the disordered porous medium is represented by a collection of $N_{\mathrm{m}}$ rigorously immobile point particles, randomly placed in a volume $V$ at positions denoted by $\mathbf{s}_{1}, \mathbf{s}_{2}, \ldots, \mathbf{s}_{N_{\mathrm{m}}}$ according to a given probability distribution $\mathcal{P}\left(\mathbf{s}_{1}, \mathbf{s}_{2}, \ldots, \mathbf{s}_{N_{\mathrm{m}}}\right)[20,21]$. Its overall density is $n_{\mathrm{m}}=N_{\mathrm{m}} / V$ and its frozen density fluctuations

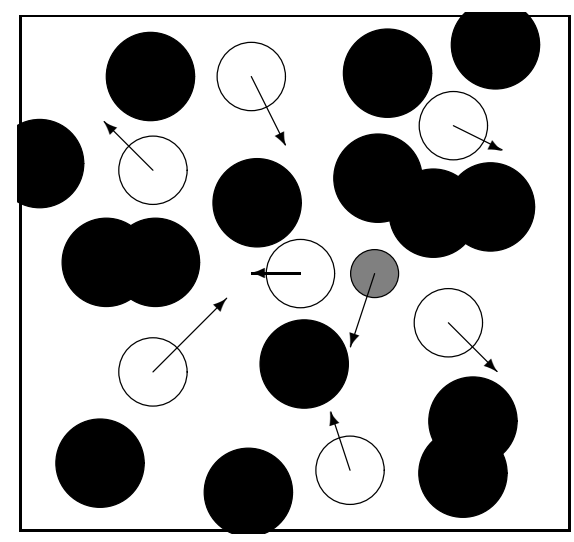

FIG. 1: Sketch of a QA system. In black, the immobile matrix particles. In white and gray, with arrows symbolizing their movement, the fluid particles and the tagged particle, respectively.

are given by

$$
\rho_{\mathbf{q}}^{\mathrm{m}}=\sum_{j=1}^{N_{\mathrm{m}}} e^{i \mathbf{q s}_{j}},
$$

where $\mathbf{q}$ denotes the wave vector.

The fluid component consists of $N_{\mathrm{f}}$ point particles (density $n_{\mathrm{f}}=N_{\mathrm{f}} / V$ ) of mass $m$, which equilibrate at a temperature $T$ in the random potential energy landscape created by the frozen matrix particles. As usual, its time-dependent density fluctuations are defined as

$$
\rho_{\mathbf{q}}^{\mathrm{f}}(t)=\sum_{j=1}^{N_{\mathrm{f}}} e^{i \mathbf{q r}_{j}(t)},
$$

where $\mathbf{r}_{j}(t)$ is the position of the fluid particle $j, j=$ $1, \ldots, N_{\mathrm{f}}$, at time $t$. But, in the case of a QA system, these quantities are not immediately useful. Indeed, because of the random external field provided by the fixed matrix, nonzero average density fluctuations exist at equilibrium. Thus, one is led to consider relaxing and non-relaxing fluid density fluctuations, corresponding to $\delta \rho_{\mathbf{q}}^{\mathrm{f}}(t)=\rho_{\mathbf{q}}^{\mathrm{f}}(t)-\left\langle\rho_{\mathbf{q}}^{\mathrm{f}}\right\rangle$ and $\left\langle\rho_{\mathbf{q}}^{\mathrm{f}}\right\rangle$, respectively, where $\langle\cdots\rangle$ denotes a thermal average taken for a given realization of the matrix.

A tagged particle of mass $m_{\mathrm{s}}$ is immersed in the system. Its time-dependent density fluctuations are given by

$$
\rho_{\mathbf{q}}^{\mathrm{s}}(t)=e^{i \mathbf{q r}^{\mathrm{s}}(t)},
$$

where $\mathbf{r}^{\mathrm{s}}(t)$ is its position at time $t$. In an infinite system, $\left\langle\rho_{\mathbf{q}}^{\mathrm{s}}\right\rangle=0$, so there is no need to separate relaxing and non-relaxing parts in this quantity.

The theory deals with disorder-averaged quantities under the assumption that the matrix is statistically homogeneous, so that, while for any single realization, the system lacks translational and rotational invariance, all 
expectation values computed with the matrix probability distribution have the same properties as in a truly translationally and rotationally invariant system. For instance, $\overline{\left\langle\rho_{\mathbf{q}}^{\mathrm{f}}\right\rangle}=0$, where $\cdots$ denotes the average over the matrix realizations performed after the thermal average $\langle\cdots\rangle$. It also results that the disorder-averaged correlation functions of all the density fluctuations present in the problem are diagonal in $\mathbf{q}$ and only depend on its modulus $q$.

The collective dynamics has been considered in Refs. [17, 18, 19]. It is described by a closed set of selfconsistent equations for the time evolution of the normalized connected autocorrelation function of the fluid density fluctuations

$$
\phi_{q}(t)=\frac{\overline{\left\langle\delta \rho_{\mathbf{q}}^{\mathrm{f}}(t) \delta \rho_{-\mathbf{q}}^{\mathrm{f}}(0)\right\rangle}}{N_{\mathrm{f}} S_{q}^{\mathrm{ff}(\mathrm{c})}},
$$

where $S_{q}^{\mathrm{ff}(\mathrm{c})}$ is the connected fluid-fluid structure factor defined as

$$
S_{q}^{\mathrm{ff}(\mathrm{c})}=\frac{1}{N_{\mathrm{f}}} \overline{\left\langle\delta \rho_{\mathbf{q}}^{\mathrm{f}} \delta \rho_{-\mathbf{q}}^{\mathrm{f}}\right\rangle} .
$$

They consist of a standard generalized Langevin equation,

$$
\ddot{\phi}_{q}(t)+\Omega_{q}^{2} \phi_{q}(t)+\Omega_{q}^{2} \int_{0}^{t} d \tau m_{q}(t-\tau) \dot{\phi}_{q}(\tau)=0,
$$

with $\Omega_{q}^{2}=q^{2} k_{B} T / m S_{q}^{\mathrm{ff}(\mathrm{c})}$ and initial conditions $\phi_{q}(0)=$ $1, \dot{\phi}_{q}(0)=0$, and of a mode-coupling approximation for the memory kernel, $m_{q}(t)=\Gamma_{q} \delta(t)+m_{q}^{(\mathrm{MC})}(t)$, where $\Gamma_{q}$ is a friction coefficient associated with fast dynamical processes and

$$
m_{q}^{(\mathrm{MC})}(t)=\int \frac{d^{3} \mathbf{k}}{(2 \pi)^{3}}\left[V_{\mathbf{q}, \mathbf{k}}^{(2)} \phi_{k}(t) \phi_{|\mathbf{q}-\mathbf{k}|}(t)+V_{\mathbf{q}, \mathbf{k}}^{(1)} \phi_{k}(t)\right],
$$

with

$$
V_{\mathbf{q}, \mathbf{k}}^{(2)}=\frac{1}{2} n_{\mathrm{f}} S_{q}^{\mathrm{ff(c)}}\left[\frac{\mathbf{q} \cdot \mathbf{k}}{q^{2}} \hat{c}_{k}^{\mathrm{ff}(\mathrm{c})}+\frac{\mathbf{q} \cdot(\mathbf{q}-\mathbf{k})}{q^{2}} \hat{c}_{|\mathbf{q}-\mathbf{k}|}^{\mathrm{ff}(\mathrm{c})}\right]^{2} S_{k}^{\mathrm{ff(c)}} S_{|\mathbf{q}-\mathbf{k}|}^{\mathrm{ff}(\mathrm{c})}
$$

and

$$
V_{\mathbf{q}, \mathbf{k}}^{(1)}=n_{\mathrm{f}} S_{q}^{\mathrm{ff}(\mathrm{c})}\left[\frac{\mathbf{q} \cdot \mathbf{k}}{q^{2}} \hat{c}_{k}{ }_{k}^{\mathrm{ff}(\mathrm{c})}+\frac{\mathbf{q} \cdot(\mathbf{q}-\mathbf{k})}{q^{2}} \frac{1}{n_{\mathrm{f}}}\right]^{2} S_{k}^{\mathrm{ff}(\mathrm{c})} S_{|\mathbf{q}-\mathbf{k}|}^{\mathrm{ff} \mid} .
$$

$S_{q}^{\mathrm{ff}(\mathrm{b})}$ is the disconnected or blocked fluid-fluid structure factor

$$
S_{q}^{\mathrm{ff(}(\mathrm{b})}=\frac{1}{N_{\mathrm{f}}} \overline{\left\langle\rho_{\mathbf{q}}^{\mathrm{f}}\right\rangle\left\langle\rho_{-\mathbf{q}}^{\mathrm{f}}\right\rangle},
$$

and $\hat{c}_{q}^{\mathrm{ff}(\mathrm{c})}$ is the Fourier transform of the connected fluidfluid direct correlation function [28, 29, 30, 31]. Its blocked counterpart will be denoted $\hat{c}_{q}^{\mathrm{ff}(\mathrm{b})}$ in the following. For reference, the relations between the different static correlation functions used in this work are reported in Appendix A.

We now turn to the tagged-particle dynamics as encoded by its density correlator

$$
\phi_{q}^{\mathrm{s}}(t)=\overline{\left\langle\rho_{\mathbf{q}}^{\mathrm{s}}(t) \rho_{-\mathbf{q}}^{\mathrm{s}}(0)\right\rangle} .
$$

Using projection operator methods, a generalized Langevin equation is first obtained,

$$
\ddot{\phi}_{q}^{\mathrm{s}}(t)+\omega_{q}^{2} \phi_{q}^{\mathrm{s}}(t)+\omega_{q}^{2} \int_{0}^{t} d \tau m_{q}^{\mathrm{s}}(t-\tau) \dot{\phi}_{q}^{\mathrm{s}}(\tau)=0,
$$

with $\omega_{q}^{2}=q^{2} k_{B} T / m_{\mathrm{s}}$ and initial conditions $\phi_{q}^{\mathrm{s}}(0)=1$, $\dot{\phi}_{q}^{\mathrm{s}}(0)=0$. This equation is exactly the same as for bulk systems.

It now remains to derive a mode-coupling approximation for the memory kernel $m_{q}^{\mathrm{s}}(t)$. Here, some care is needed. Indeed, it was found in Ref. [19] that, in the case of an isolated molecule moving in a random porous matrix, a direct calculation based on the fact that the molecule interacts with the solid only [25, 26, 27] does not yield the same result as taking the limit of vanishing fluid density in the equations describing the collective dynamics, while the two approaches should be equivalent. The present problem, of which the above is actually a special case, shows a similar difficulty. A direct approach in which the tagged-particle density fluctuations only couple to the matrix and fluid density fluctuations results in different equations as considering an adsorbed binary fluid mixture in the limit of a vanishing concentration of one of the components. In the absence of any obvious physical reason to consider the zero concentration case as a special one, we make the choice of consistency at all densities and use the latter strategy. Additional arguments in favor of this choice and the equations obtained with the alternative approach are reported in the next section.

So we start with the MCT equations for a mixture adsorbed in a disordered porous matrix, which are given for completeness in appendix B, specialize them to the se of a binary mixture, and send the concentration of e component to zero. Keeping only the leading terms, we get $m_{q}^{\mathrm{s}}(t)=\Gamma_{q}^{\mathrm{s}} \delta(t)+m_{q}^{\mathrm{s}(\mathrm{MC})}(t)$, where here again $\Gamma_{q}^{\mathrm{s}}$ is a friction coefficient associated with fast dynamical processes and

$$
m_{q}^{\mathrm{s}(\mathrm{MC})}(t)=\int \frac{d^{3} \mathbf{k}}{(2 \pi)^{3}}\left[v_{\mathbf{q}, \mathbf{k}}^{(2)} \phi_{k}^{\mathrm{s}}(t) \phi_{|\mathbf{q}-\mathbf{k}|}(t)+v_{\mathbf{q}, \mathbf{k}}^{(1)} \phi_{k}^{\mathrm{s}}(t)\right],
$$

with

$$
v_{\mathbf{q}, \mathbf{k}}^{(2)}=n_{\mathrm{f}}\left[\frac{\mathbf{q} \cdot(\mathbf{q}-\mathbf{k})}{q^{2}}\right]^{2}\left[\hat{c}_{|\mathbf{q}-\mathbf{k}|}^{\mathrm{ff}(\mathrm{c})}\right]^{2} S_{|\mathbf{q}-\mathbf{k}|}^{\mathrm{ff(c)}},
$$

and

$$
v_{\mathbf{q}, \mathbf{k}}^{(1)}=\left[\frac{\mathbf{q} \cdot(\mathbf{q}-\mathbf{k})}{q^{2}}\right]^{2} \hat{h}_{|\mathbf{q}-\mathbf{k}|}^{\mathrm{ss} \mid} .
$$


$\hat{c}_{q}^{\mathrm{sf}(\mathrm{c})}$ and $\hat{h}_{q}^{\mathrm{ss}(\mathrm{b})}$ are the Fourier transforms of the connected single-particle-fluid direct correlation function and of the single-particle-single-particle blocked total correlation function, respectively (see Ref. [32] for general definitions). They obviously have blocked and connected counterparts, denoted by $\hat{c}_{q}^{\mathrm{sf}(\mathrm{b})}$ and $\hat{h}_{q}^{\mathrm{ss}(\mathrm{c})}$, respectively.

Like in the bulk, the last set of equations is not closed: one first needs to solve the collective dynamics for $\phi_{q}(t)$ before one can compute $\phi_{q}^{\mathrm{s}}(t)$. As it should, in the $n_{\mathrm{m}} \rightarrow 0$ and $n_{\mathrm{f}} \rightarrow 0$ limits, the equations for the taggedparticle dynamics in bulk systems [14] and those for the collective dynamics in the zero fluid density limit [19] are recovered, respectively. The same remarks as in Ref. [19] apply: the presence of a linear term in the memory kernel, the disappearance of any explicit reference to the matrix, the presence of connected and disconnected structural quantities only, and the fact that $v_{\mathbf{q}, \mathbf{k}}^{(2)}$ is the same as in a bulk system, with connected quantities simply replacing the fluid structure factor and direct correlation function. We refer the reader to the previous work for the corresponding discussion.

Finally, from the above formulas, one can derive an equation for the time evolution of the mean-squared displacement $\delta r^{2}(t)=\overline{\left\langle\left|\mathbf{r}^{\mathbf{s}}(t)-\mathbf{r}^{\mathbf{s}}(0)\right|^{2}\right\rangle}$, by using the small wave vector behavior of the tagged-particle density correlation function, $\phi_{q}^{\mathrm{s}}(t)=1-q^{2} \delta r^{2}(t) / 6+O\left(q^{4}\right)$. It reads [note that it is possible to integrate once, thanks to the initial conditions $\delta r^{2}(0)=0$ and $\left.\dot{\delta \dot{r}^{2}}(0)=0\right]$

$$
\dot{\delta \dot{r}^{2}}(t)+\frac{k_{B} T}{m_{\mathrm{s}}} \int_{0}^{t} d \tau m^{\mathrm{MSD}}(t-\tau) \delta r^{2}(\tau)=\frac{6 k_{B} T}{m_{\mathrm{s}}} t
$$

with $m^{\mathrm{MSD}}(t)=\lim _{q \rightarrow 0} q^{2} m_{q}^{\mathrm{s}}(t)=\gamma \delta(t)+m^{\mathrm{MSD}(\mathrm{MC})}(t)$, where $\gamma=\lim _{q \rightarrow 0} q^{2} \Gamma_{q}^{\mathrm{s}}$ relates to the fast dynamical processes and where the mode-coupling part of the memory kernel is given by

$$
m^{\mathrm{MSD}(\mathrm{MC})}(t)=\int_{0}^{+\infty} \frac{k^{4} d k}{6 \pi^{2}}\left[w_{k}^{(2)} \phi_{k}^{\mathrm{s}}(t) \phi_{k}(t)+w_{k}^{(1)} \phi_{k}^{\mathrm{s}}(t)\right]
$$

with

$$
w_{k}^{(2)}=n_{\mathrm{f}}\left[\hat{c}_{k}^{\mathrm{sf}(\mathrm{c})}\right]^{2} S_{k}^{\mathrm{ff}(\mathrm{c})} \text { and } w_{k}^{(1)}=\hat{h}_{k}^{\mathrm{ss}(\mathrm{b})} .
$$

\section{CONNECTIONS WITH PREVIOUS THEORIES AND A VARIANT}

The physics of QA mixtures involves a number of subtleties, related, for instance, to the splitting of the static correlations into connected and disconnected parts. These might induce special difficulties and put constraints on the proper way to set up a dynamical theory for these systems. In this technical section, we illustrate how they manifest themselves within the MCT framework, in an attempt to provide guidelines for future work based on other formalisms (see Ref. [33], for instance).
In Ref. [19], the MCT equations for the collective dynamics in a QA binary mixture have been compared to those for the residual dynamics of a bulk system in its ideal glassy phase. Strong analogies have been found in the overall structure of both theories, which originate in the presence of time-persistent density fluctuations in both cases. But there are significant and irreducible differences as well, which reflect the different origins of these frozen fluctuations, static and disorder-induced in the former case, dynamical and self-induced in the latter.

The main conclusion from this study was that, in general, it does not seem sensible to try and derive a dynamical theory for QA systems by simply taking the limit of the corresponding approach for fully annealed mixtures in which one component representing the solid matrix would become immobile, or at least extreme care should be taken. Since this contradicts naive expectations, we begin the present section with a similar analysis of the MCT for the tagged-particle dynamics. This is clearly a simpler problem, since by construction no collective dynamical phenomena come into play and there is no disconnected component in the tagged-particle density correlation function.

In order to complete the discussion of Ref. [19], we first consider the case of a one-component bulk fluid in its ideal glassy phase. The tagged-particle dynamics as expressed in terms of the residual relaxation of the fluid density fluctuations obeys mode-coupling equations of the same form as above, with vertices [14]

$$
v_{\mathbf{q}, \mathbf{k}}^{(2)}=n_{\mathrm{f}}\left[\frac{\mathbf{q} \cdot(\mathbf{q}-\mathbf{k})}{q^{2}}\right]^{2}\left[\hat{c}_{|\mathbf{q}-\mathbf{k}|}^{\mathrm{sf}}\right]^{2}\left\{\left(1-f_{|\mathbf{q}-\mathbf{k}|}\right) S_{|\mathbf{q}-\mathbf{k}|}^{\mathrm{ff}}\right\},
$$

and

$$
v_{\mathbf{q}, \mathbf{k}}^{(1)}=n_{\mathrm{f}}\left[\frac{\mathbf{q} \cdot(\mathbf{q}-\mathbf{k})}{q^{2}}\right]^{2}\left[\hat{c}_{|\mathbf{q}-\mathbf{k}|}^{\mathrm{sf}}\right]^{2}\left\{f_{|\mathbf{q}-\mathbf{k}|} S_{|\mathbf{q}-\mathbf{k}|}^{\mathrm{ff}}\right\},
$$

where $n_{\mathrm{f}}$ is the density of the fluid, $S_{q}^{\mathrm{ff}}$ its structure factor, $\hat{c}_{q}^{\text {sf }}$ the Fourier transform of the single-particle-fluid direct correlation function, and $f_{q}$ the Debye-Waller factor of the glass. To make contact with the theory for QA systems, we now need an expression for $\hat{h}_{q}^{\text {ss(b) }}$ in Eq. (13b). Since the present problem corresponds to a situation where there are fluid density fluctuations only, we shall take advantage of the fact that the matrix does not appear explicitly in the MCT equations for QA systems and use the Ornstein-Zernike (OZ) equations for a simpler example of a fluid in a random environment, namely, a fluid plunged in a Gaussian random field [34]. One then gets $[35,36]$

$$
\hat{h}_{q}^{\mathrm{ss}(\mathrm{b})}=\hat{c}_{q}^{\mathrm{ss}(\mathrm{b})}+2 n_{\mathrm{f}} \hat{c}_{q}^{\mathrm{ff}(\mathrm{b})} \hat{c}_{q}^{\mathrm{sf}(\mathrm{c})} S_{q}^{\mathrm{ff}(\mathrm{c})}+n_{\mathrm{f}}\left[\hat{c}_{q}^{\mathrm{ff}(\mathrm{c})}\right]^{2} S_{q}^{\mathrm{ff}(\mathrm{b})},
$$

where $\hat{c}_{q}^{\mathrm{ss}(\mathrm{b})}$ is the Fourier transform of the single-particlesingle-particle blocked direct correlation function. Thus, we observe that there is an exact correspondence between 
the two theories, with the same role played by $S_{q}^{\mathrm{ff}(\mathrm{c})}$ and $\left(1-f_{q}\right) S_{q}^{\mathrm{ff}}$ on the one hand, $S_{q}^{\mathrm{ff}(\mathrm{b})}$ and $f_{q} S_{q}^{\mathrm{ff}}$ on the other hand, provided one disregards in Eq. (18) the terms involving blocked direct correlation functions. This in particular insures that the full and connected direct correlation functions coincide.

As a second step, let us now approach the problem following the empirical strategy outlined above, i.e., we shall consider the QA binary mixture as a special limit of a fully annealed binary mixture. As already pointed out, this approach is ineffective for the description of the collective dynamics [19]. For a tagged particle moving in a fully annealed binary mixture, the MCT result for the memory kernel reads (for simplicity, we keep the same species labels as in the QA binary mixture) [22, 24, 37]

$$
\begin{gathered}
m_{q}^{\mathrm{s}(\mathrm{MC})}(t)=\left(n_{\mathrm{f}}+n_{\mathrm{m}}\right) \int \frac{d^{3} \mathbf{k}}{(2 \pi)^{3}}\left[\frac{\mathbf{q} \cdot(\mathbf{q}-\mathbf{k})}{q^{2}}\right]^{2} \phi_{k}^{\mathrm{s}}(t) \\
\left\{\left[\hat{c}_{|\mathbf{q}-\mathbf{k}|}^{\mathrm{sf}}\right]^{2} F_{|\mathbf{q}-\mathbf{k}|}^{\mathrm{ff}}(t)+2 \hat{c}_{|\mathbf{q}-\mathbf{k}|}^{\mathrm{sf}} \hat{c}_{|\mathbf{q}-\mathbf{k}|}^{\mathrm{sm}} F_{|\mathbf{q}-\mathbf{k}|}^{\mathrm{fm}}(t)\right. \\
\left.+\left[\hat{c}_{|\mathbf{q}-\mathbf{k}|}^{\mathrm{sm}}\right]^{2} F_{|\mathbf{q}-\mathbf{k}|}^{\mathrm{mm}}(t)\right\},
\end{gathered}
$$

where $\hat{c}_{q}^{\mathrm{sm}}$ is the Fourier transform of the single-particlematrix direct correlation function and, in the definitions of the density correlation functions $F_{q}^{\mathrm{ff}}(t), F_{q}^{\mathrm{fm}}(t)$, and $F_{q}^{\mathrm{mm}}(t)$, the usual normalization for binary mixtures has been used, hence the prefactor $n_{\mathrm{f}}+n_{\mathrm{m}}$. We might now adapt this equation to the case of a QA binary mixture, for which $F_{q}^{\mathrm{ff}}(t)$ splits into relaxing and frozen parts according to

$$
\left(n_{\mathrm{f}}+n_{\mathrm{m}}\right) F_{q}^{\mathrm{ff}}(t) \equiv n_{\mathrm{f}} S_{q}^{\mathrm{ff(}(\mathrm{b})}+n_{\mathrm{f}} S_{q}^{\mathrm{ff(c})} \phi_{q}(t),
$$

while $F_{q}^{\mathrm{fm}}(t)$ and $F_{q}^{\mathrm{mm}}(t)$ are actually time-independent and given by

$$
\begin{gathered}
\left(n_{\mathrm{f}}+n_{\mathrm{m}}\right) F_{q}^{\mathrm{fm}}(t) \equiv \sqrt{n_{\mathrm{f}} n_{\mathrm{m}}} S_{q}^{\mathrm{fm}}, \\
\left(n_{\mathrm{f}}+n_{\mathrm{m}}\right) F_{q}^{\mathrm{mm}}(t) \equiv n_{\mathrm{m}} S_{q}^{\mathrm{mm}} .
\end{gathered}
$$

$S_{q}^{\mathrm{fm}}$ and $S_{q}^{\mathrm{mm}}$ are the fluid-matrix and matrix-matrix structure factors, respectively, and, like $S_{q}^{\mathrm{ff}(\mathrm{b})}$ and $S_{q}^{\mathrm{ff(c)}}$, they are defined with the normalization suitable for QA mixtures (see Appendix A). One then obtains modecoupling equations of the same form as above, with vertices

$$
v_{\mathbf{q}, \mathbf{k}}^{(2)}=n_{\mathrm{f}}\left[\frac{\mathbf{q} \cdot(\mathbf{q}-\mathbf{k})}{q^{2}}\right]^{2}\left[\hat{c}_{|\mathbf{q}-\mathbf{k}|}^{\mathrm{sf}}\right]^{2} S_{|\mathbf{q}-\mathbf{k}|}^{\mathrm{ff}(\mathrm{c})},
$$

and

$$
\begin{aligned}
& v_{\mathbf{q}, \mathbf{k}}^{(1)}=\left[\frac{\mathbf{q} \cdot(\mathbf{q}-\mathbf{k})}{q^{2}}\right]^{2}\left(n_{\mathrm{f}}\left[\hat{c}_{|\mathbf{q}-\mathbf{k}|}^{\mathrm{sf}}\right]^{2} S_{|\mathbf{q}-\mathbf{k}|}^{\mathrm{ff}(\mathrm{b})}\right. \\
& \left.+2 \sqrt{n_{\mathrm{f}} n_{\mathrm{m}}} \hat{c}_{|\mathbf{q}-\mathbf{k}|}^{\mathrm{sf}} \hat{c}_{|\mathbf{q}-\mathbf{k}|}^{\mathrm{sm}} S_{|\mathbf{q}-\mathbf{k}|}^{\mathrm{fm}}+n_{\mathrm{m}}\left[\hat{c}_{|\mathbf{q}-\mathbf{k}|}^{\mathrm{sm}}\right]^{2} S_{|\mathbf{q}-\mathbf{k}|}^{\mathrm{mm}}\right) .
\end{aligned}
$$

If this result is confronted to the relevant expression of $\hat{h}_{q}^{\mathrm{ss}(\mathrm{b})}[32]$,

$$
\begin{aligned}
\hat{h}_{q}^{\mathrm{ss}(\mathrm{b})}= & \hat{c}_{q}^{\mathrm{ss}(\mathrm{b})}+2 n_{\mathrm{f}} \hat{c}_{q}^{\mathrm{ff}(\mathrm{b})} \hat{c}_{q}^{\mathrm{sf}(\mathrm{c})} S_{q}^{\mathrm{ff}(\mathrm{c})}+n_{\mathrm{f}}\left[\hat{c}_{q}^{\mathrm{ff}(\mathrm{c})}\right]^{2} S_{q}^{\mathrm{ff}(\mathrm{b})} \\
& +2 \sqrt{n_{\mathrm{f}} n_{\mathrm{m}}} \hat{c}_{q}^{\mathrm{ff}(\mathrm{c})} \hat{c}_{q}^{\mathrm{sm}} S_{q}^{\mathrm{fm}}+n_{\mathrm{m}}\left[\hat{c}_{q}^{\mathrm{sm}}\right]^{2} S_{q}^{\mathrm{mm}}, \quad(22)
\end{aligned}
$$

an exact correspondence with equations (13) is once again found, under the same conditions as in the previous calculation.

So, we find that, in both cases, there would be a perfect consistency between the derived equations and the theory developed for fluids in random environments if all blocked direct correlation functions were identically zero. Or, stated differently, both approaches would be perfectly acceptable starting points for heuristic derivations of the present theory if this condition was fulfilled. Obviously, it is not, and this provides further arguments in favor of a differentiated treatment of fully annealed and quenched-annealed mixtures.

However, at the same time, the approximation which consists in neglecting the blocked direct correlation functions is a very common one, which for historical reasons is often referred to as the Madden-Glandt approximation $[28,29,30]$. Its widespread use originates in the fact that these functions are among the specific features of fluids in quenched disordered environments which are the most difficult to capture with simple approximations, and, for instance, many standard closures of the replica OZ equations erroneously prescribe that they vanish identically $[28,29,30,38]$. It is thus hardly surprising that they appear in the errors made when trying to infer a theory for QA systems starting from a similar approach for fully annealed systems, for which these functions are meaningless.

The crucial simplification in the case of the taggedparticle dynamics, compared to the collective dynamics, is that the Madden-Glandt approximation seems to be the only required approximation [39]. This is explicitly demonstrated here in the framework of the MCT, but we expect that analogous situations could occur with other theoretical schemes. So, the conclusion could be that it does not appear unreasonable to try and derive empirical theories for the tagged-particle dynamics in QA systems starting from approaches developed for fully equilibrated systems, but this will usually be at the cost of additional implicit approximations like Madden-Glandt's. Fortunately, most of the time, such approximations have quantitative consequences only and the cost appears modest. Note however that one serious restriction remains: since no such simple empirical scheme seems to be generically applicable to the collective dynamics [19], it will always be difficult to give a proper account of the situations in which this part of the dynamics matters, for instance, if the coupling of the single-particle and collective dynamics is strong.

Another illustration of the subtle interplay between the dynamical theory and the peculiar structure of the static 
correlations in fluids evolving in random environments is provided by the alternative derivation of the MCT for the tagged-particle dynamics in QA systems which has been discarded in the previous section. It is based on the observation that the only forces exerted on the tagged molecule are those due to the fluid and the random matrix. So, on simple physical grounds, one expects that the only mode-coupling contributions to the relaxation kernel will come from the products $\rho_{\mathbf{k}}^{\mathrm{s}} \delta \rho_{\mathbf{q}-\mathbf{k}}^{\mathrm{f}}, \rho_{\mathbf{k}}^{\mathrm{s}}\left\langle\rho_{\mathbf{q}-\mathbf{k}}^{\mathrm{f}}\right\rangle$, and $\rho_{\mathbf{k}}^{\mathrm{s}} \rho_{\mathbf{q}-\mathbf{k}}^{\mathrm{m}}$. Projecting the random forces on this set of variables using standard projection operator methods, one obtains a memory kernel which is essentially the same as above, except that one has to replace in Eq. (13b) $\hat{h}_{|\mathbf{q}-\mathbf{k}|}^{\mathrm{ss}(\mathrm{b})}$ as given by Eq. (22) by $\hat{h}_{|\mathbf{q}-\mathbf{k}|}^{\mathrm{ss}(\mathrm{b})}-\hat{c}_{|\mathbf{q}-\mathbf{k}|}^{\mathrm{ss}(\mathrm{b})}+\left[\hat{c}_{|\mathbf{q}-\mathbf{k}|}^{\mathrm{sf}(\mathrm{b})}\right]^{2} / \hat{c}_{|\mathbf{q}-\mathbf{k}|}^{\mathrm{ff}(\mathrm{b})}$.

An immediate reason to reject this approach is that, in the limit $n_{\mathrm{f}} \rightarrow 0$ where the dynamics should be determined by the properties of the matrix and of the tagged particle only, an unphysical dependence on the fluid would remain in the theory because of the last term of this expression. This is avoided with the derivation retained in the previous section, which is thus clearly preferable.

The only difference between the two approaches is that, by first working with a finite concentration of tagged particles, the correct one incorporates contributions associated with their frozen density fluctuations, which seem to be preserved when the concentration is sent to zero. In this respect, it is pictorial that the two approaches happen to agree when the fluid and tagged particles are identical, thanks to the equalities $\hat{c}_{q}^{\mathrm{ss}(\mathrm{b})}=\hat{c}_{q}^{\mathrm{sf}(\mathrm{b})}=\hat{c}_{q}^{\mathrm{ff}(\mathrm{b})}$. Indeed, it is in this unique case that the frozen fluid and tagged-particle density fluctuations share the same statistics and that the former can act in the incorrect approach as substitutes for the latter which would otherwise be missing. Thus, the inclusion of contributions due to the frozen tagged-particle density fluctuations, which are rather elusive as far as species with vanishing concentration are concerned, appears as an important requirement in order to derive an acceptable theory. Retrospectively, failure to take into account similar contributions in special limiting cases (vanishing fluid density, adsorbed ideal gas) of the collective dynamics surely explains some ambiguous results pointed out in Ref. [19]. At present, the physics, if any, behind this constraint is unclear.

\section{DYNAMICAL PHASE DIAGRAMS}

We now turn to the quantitative results of the theory, which require numerical solutions of the MCT equations. In the present section, we complete the dynamical phase diagrams of Ref. [19], where the liquid-glass transition lines for two simple model systems had been determined, while, in the next one, the full dynamical scenarios for the tagged-particle motion will be discussed.

The systems to be considered are two closely related QA mixture models in which both the fluid-fluid and fluid-matrix interactions are pure hard core repulsions of the same diameter $d$. The only difference between them lies in the matrix correlations. In model $\mathrm{I}$, the matrix particles are not allowed to overlap, so that the configurational statistics of the porous medium is that of an equilibrium hard sphere fluid, while in model II the matrix particles are completely uncorrelated and overlap freely, as in an ideal gas. For both systems, the two dimensionless densities $\phi_{\mathrm{f}}=\pi n_{\mathrm{f}} d^{3} / 6$ and $\phi_{\mathrm{m}}=\pi n_{\mathrm{m}} d^{3} / 6$ will be used as control parameters, and the structural quantities required by the dynamical theory will be computed with the Percus-Yevick (PY) approximation [28, 29, 30, 38].

For simplicity, we shall concentrate on the case where the tagged particle is one of the fluid particles, so that in Eqs. (13) and (16) one has to take $\hat{c}_{q}^{\mathrm{sf}(\mathrm{c})}=\hat{c}_{q}^{\mathrm{ff}(\mathrm{c})}$ and $\hat{h}_{q}^{\mathrm{ss}(\mathrm{b})}=\hat{h}_{q}^{\mathrm{ff}(\mathrm{b})}$. Then, the tagged-particle dynamics coincides with the self part of the collective dynamics.

From the point of view of the single-particle dynamics, two distinct phases exist in MCT. If for all $q$ $\lim _{t \rightarrow \infty} \phi_{q}^{\mathbf{s}}(t)=0$, one is in the diffusive regime, where the tagged particle can explore an infinite domain and move infinitely far away from its initial position. If on the contrary for all $q \lim _{t \rightarrow \infty} \phi_{q}^{\mathrm{s}}(t)=f_{q}^{\mathrm{s}} \neq 0$, one is in the localized regime, where the tagged particle is trapped in a finite domain.

The single-particle nonergodicity parameter $f_{q}^{\mathrm{s}}$, which is usually called the Lamb-Mössbauer factor, is the solution of the nonlinear set of equations

$$
\frac{f_{q}^{\mathrm{s}}}{1-f_{q}^{\mathrm{s}}}=\int \frac{d^{3} \mathbf{k}}{(2 \pi)^{3}}\left[v_{\mathbf{q}, \mathbf{k}}^{(2)} f_{k}^{\mathrm{s}} f_{|\mathbf{q}-\mathbf{k}|}+v_{\mathbf{q}, \mathbf{k}}^{(1)} f_{k}^{\mathrm{s}}\right]
$$

where $f_{q}$ is the collective dynamics nonergodicity parameter or Debye-Waller factor computed in Ref. [19]. This equation has to be solved numerically in order to locate the diffusive and localized phases when $\phi_{\mathrm{f}}$ and $\phi_{\mathrm{m}}$ are varied. All computations in the present paper have been achieved using the methods of Refs. [40, 41], to which the interested reader is referred for technical details, with the same parameters as in Ref. [19]. As pointed out in the latter work, the discretization used for the wave vector integration in Eq. (23) involves a cutoff of the low $q$ divergence of the memory kernels. This has the advantage of eliminating spurious long time anomalies originating in this divergence [42, 43, 44], but at the cost of quantitative uncertainties due to the arbitrariness of the cutoff value. They have been estimated in two ways, first by test calculations on a finer $q$ grid, second by a comparison with the results obtained within an additional hydrodynamic approximation (see Appendix $\mathrm{C}$ ) which allows one to integrate exactly over the full $q$ range [26]. It has been found that the errors on the location of the transition points never exceed a few percents.

The dynamical phase diagrams of models I and II are reported in Fig. 2. The liquid-glass transition lines of types A and B are those obtained in Ref. [19]. As discussed there, for model I they join smoothly at a degenerate higher-order $A_{3}$ singularity, while for model II a 

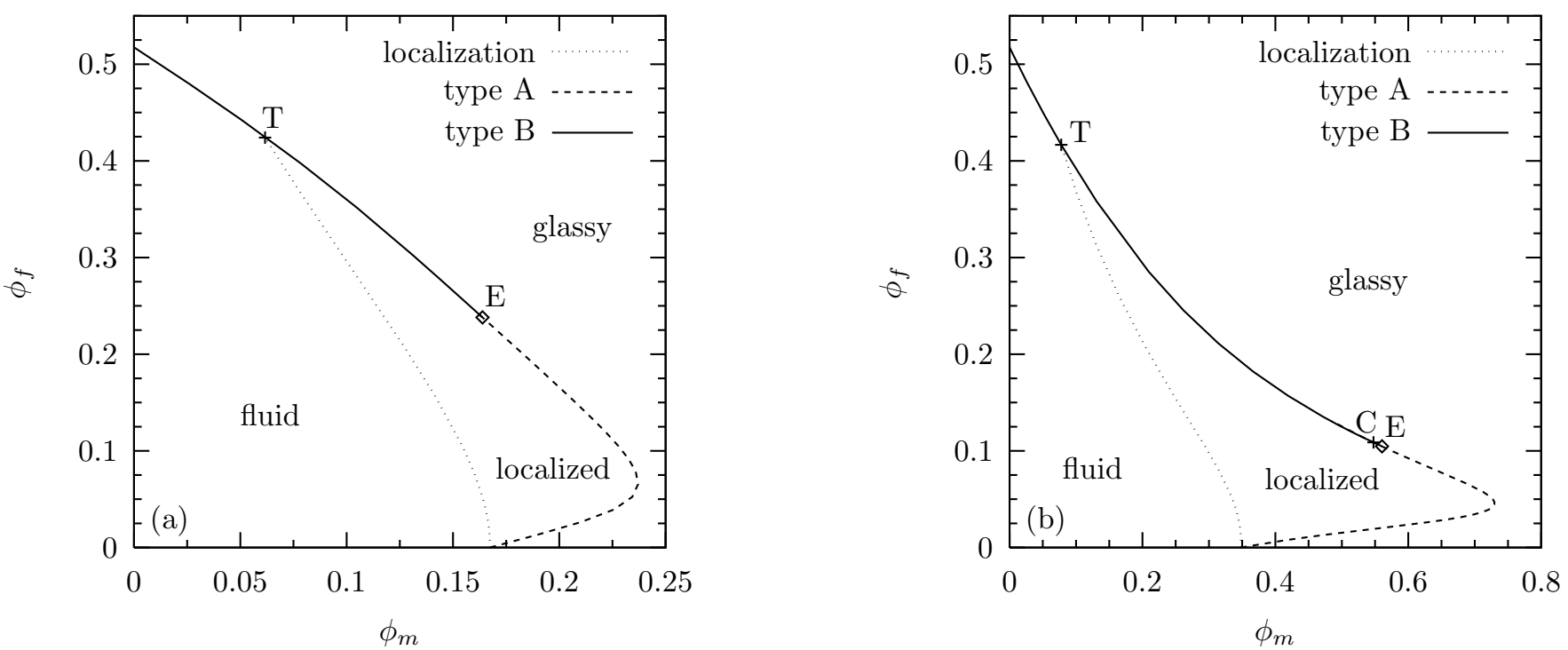

FIG. 2: Dynamical phase diagrams of two types of hard-sphere quenched-annealed binary mixtures. (a) Model I: nonoverlapping matrix particles. (b) Model II: freely overlapping matrix particles. For the collective dynamics, the $A_{3}$ endpoint singularities are denoted by $\mathrm{E}$ and the crossing point between the type A and B transition lines in model II is denoted by C. For the tagged-particle dynamics, the crossing points between the diffusion-localization and glass transition lines are denoted by $\mathrm{T}$ (for triple point).

crossing point followed by a line of glass-glass transitions terminating at an ordinary $A_{3}$ singularity is obtained. Except for these details, both phase diagrams are very similar at the level of the collective dynamics.

This remains true when the tagged-particle dynamics is considered. For both models, two types of transitions between the diffusive and localized phases are found.

At low matrix densities, the glass and localization transitions occur simultaneously. In fact, it is the spontaneous arrest of the collective dynamics which triggers the arrest of the tagged-particle dynamics through their coupling in the bilinear term of the memory kernel (12). This is the well known scenario found in all MCT studies of one-component bulk systems so far, in which the tagged-particle dynamics inherits its discontinuous (type B) character and quantitative features (critical laws, exponent parameter $\lambda$ ) from the collective dynamics $[14,41]$. Its extension to weakly confined systems is easily understood from simple continuity arguments in the vicinity of the bulk transition at $\phi_{\mathrm{m}}=0$.

These arguments break down at higher matrix densities, where one finds that the tagged particles are already localized when the ideal glass transition occurs. Thus, the latter only manifests itself as a secondary dynamical transition in an already arrested single-particle dynamics, with characteristic features imposed by the collective dynamics just like in the previous scenario. In fact, in this density domain, the liquid-glass transition is always preceded by a continuous diffusion-localization transition driven by the linear term of the memory kernel (12). These transitions, which only manifest themselves in the single-particle dynamics, are special cases of type A tran- sitions, characterized by an exponent parameter $\lambda=0$, like the pure diffusion-localization transition at $\phi_{\mathrm{f}}=0$ $[19,25,26]$. Similar ones have been previously discussed in the framework of schematic models [14, 45, 46, 47], and, more interestingly, have also been found in early MCT studies of bulk binary hard sphere mixtures with large size asymmetry, where they take place in the ideal glassy phase and correspond to the localization of the smaller species in the voids of the glass [22, 23, 24]. Superficially, the analogy between the two systems can be understood by seeing the disordered porous solid in the QA mixture as a glassy component or by considering the induction of permanent fluid density fluctuations by the quenched random environment as some kind of previtrification. But we stress once again that, as illustrated in Ref. [19] and in the previous section, such simple pictures do not in general lend themselves to the development of rigorous approaches to the dynamics of QA systems.

As seen in Fig. 2, the corresponding transition line starts on the $\phi_{\mathrm{f}}=0$ axis at the same point as the liquidglass transition line, since in this limit the collective dynamics exactly reduces to the single-particle dynamics. As $\phi_{\mathrm{f}}$ is increased, it runs across the fluid domain as obtained from the study of the collective dynamics, and it terminates when it intercepts the type B liquid-glass transition line at some finite value of $\phi_{\mathrm{m}}$.

The final result is thus that the dynamical phase diagrams of both QA mixture models display three domains, as shown in Fig. 2. For low overall densities, the dynamics is fluid-like, with both the collective and taggedparticle density fluctuations relaxing to their equilibrium values at long times. For intermediate densities, the dy- 
namics is localized, with a complete relaxation of the collective density fluctuations only. And for high densities, glassy dynamics is found, in which neither the collective nor the single-particle density fluctuations do return to equilibrium. These three domains meet at two points: first, at the pure diffusion-localization transition point at $\phi_{\mathrm{f}}=0$, because, as mentioned above, there coincide the collective and tagged-particle dynamics; second, at the crossing point between the liquid-glass and continuous diffusion-localization transition lines. For definiteness, because of the three phases, we shall call this point a triple point denoted by $\mathrm{T}$ in Fig. 2. But it should be remembered that phase coexistence is excluded in MCT, because of a maximum property of the physical solution of the MCT equations [14]. It results that point $\mathrm{T}$ corresponds unambiguously to a glassy state.

The clear separation between the diffusion-localization and liquid-glass transition lines allows one to completely disregard the collective dynamics when dealing with the asymptotic properties of the tagged-particle dynamics in the vicinity of the former transition. This possibility is particularly interesting if an additional hydrodynamic approximation is performed, since significant analytic progress can then be made in the study of the continuous diffusion-localization transition. This approach, which extends Leutheusser's theory [26] to finite fluid densities, is discussed in Appendix C.

Beside the nature of the domains and transition lines met in the phase diagrams, their shape is of interest as well. A remarkable result of Ref. [19] has been the prediction of a reentry phenomenon in the liquid-glass transition line for high matrix-low fluid densities, which has been interpreted as the signature of a dynamical decorrelation mechanism due to fluid-fluid collisions competing with the reduction of free volume and the localization effect due to the disordered porous solid. A similar feature, which might be understood using the same physical arguments, is also present in the continuous diffusionlocalization transition line, but with a much weaker amplitude, so that one needs the strong magnifications of Fig. 3 in order to visualize it.

As already pointed out in Ref. [19], a word of caution is needed about this finding. Indeed, for systems ruled by hard core fluid-matrix interactions like those studied in the present work, a number of recent extensive simulation studies $[48,49,50,51,52,53,54]$ have clearly demonstrated that the localization transition is actually driven by the percolation transition of the matrix void space, i.e., localization occurs because, above a certain critical matrix density, the void space only consists of finite disconnected domains. Such a scenario definitely rules out the possibility of a reentry phenomenon in the diffusion-localization transition line as obtained above. Indeed, if at a given value of $\phi_{\mathrm{m}}$ a system with $\phi_{\mathrm{f}}=0$ is localized because of the onset of the percolation transition, any system with a finite $\phi_{\mathrm{f}}$ at the same matrix density will be localized as well, since a variation of $\phi_{\mathrm{f}}$ obviously has no effect on the topology of the matrix
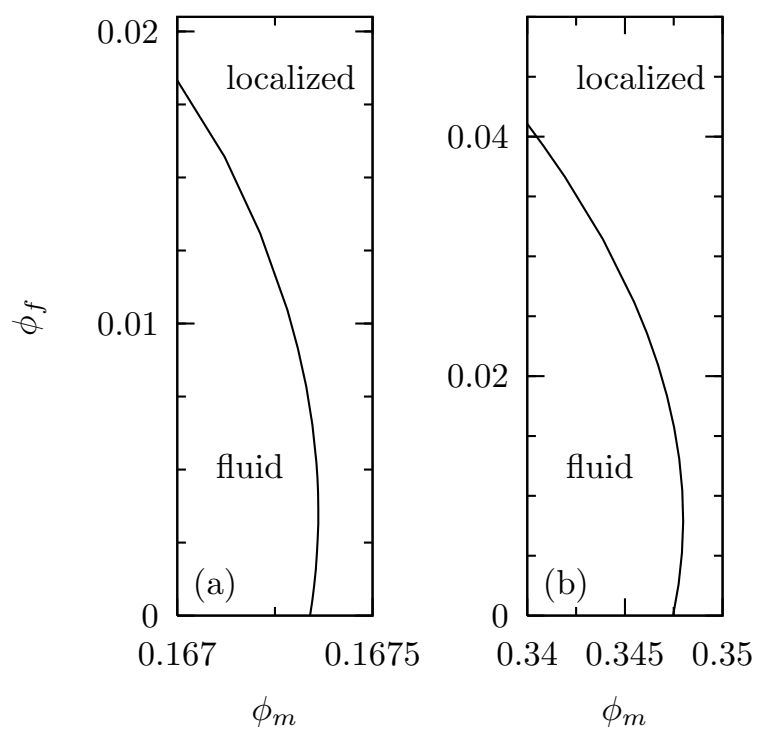

FIG. 3: Magnified views of the continuous diffusionlocalization transition lines for high matrix densities. A reentrant behavior is clearly visible. (a) Model I, (b) Model II.

\section{empty space.}

Fortunately, the amplitude of the reentry phenomenon predicted by the MCT for the diffusion-localization transition is very small, so the inconsistency is quantitatively not too serious, but, clearly, this prediction should not be taken literally. Instead, a reasonable expectation is typically a nonmonotonic variation of the self-diffusion coefficient in the slowly diffusive regime just below the localization threshold, with first an increase due to fluidfluid collisions when increasing $\phi_{\mathrm{f}}$ from zero. In support of this suggestion, we recall that, in a number of systems for which the MCT has predicted reentrant transition lines, such a reentry has been observed in the isodiffusivity curves as obtained by molecular dynamics simulations $[55,56,57,58,59,60,61]$. The same behavior should also be expected in systems with soft repulsive fluid-matrix interactions, for which the percolation concepts are only approximate. In this respect, it is encouraging that this is precisely what has been observed in a computer simulation study of a two-dimensional lattice gas model with fixed randomly placed penetrable scatterers [62].

\section{DYNAMICAL SCENARIOS}

According to the phase diagrams reported in the previous section, both QA mixtures considered in this work display three generic types of global dynamical scenarios in response to increases of the overall density of the fluidmatrix system. For small $\phi_{\mathrm{m}}$, a bulk-like type B ideal glass transition scenario prevails, in which both the collective and single-particle density fluctuations simultaneously show discontinuities. For moderate $\phi_{\mathrm{m}}$, a continuous diffusion-localization transition first occurs, followed 
at a higher density by a type B ideal glass transition. Finally, for high $\phi_{\mathrm{m}}$, the latter transition is of type A.

It is the purpose of this section to provide illustrations of these three typical scenarios and to discuss their features which might be relevant for comparisons of experimental or simulation data with the predictions of the theory. As in Ref. [19], the analytic asymptotic results shall be quoted without their proofs, which can be found in or adapted from Ref. [14] (see also Ref. [47] for type A transitions). Note that these results, which are obtained under the assumption of regular vertices, as well as the method of solution of the mode-coupling equations used in this work [40,41], which involves a cutoff of the memory kernels at low $q$, cannot give an account of possible dynamical features originating in the divergence of the mode-coupling vertices when $q \rightarrow 0$ [42, 43, 44]. While this might look unsatisfactory from a mathematical point of view, such an approximation is in fact required for physical reasons, since the low $q$ singularity is actually known to be an ill feature of the mode-coupling approximation which results in spurious long time anomalies [43].

For simplicity and consistency with Ref. [19], we follow Refs. [40] and [41] and consider the dynamics in the overdamped limit valid for Brownian systems. The generalized Langevin equations (11) and (14) then reduce to

$$
\tau_{q}^{\mathrm{s}} \dot{\phi}_{q}^{\mathrm{s}}(t)+\phi_{q}^{\mathrm{s}}(t)+\int_{0}^{t} d \tau m_{q}^{\mathrm{s}(\mathrm{MC})}(t-\tau) \dot{\phi}_{q}^{\mathrm{s}}(\tau)=0
$$

and

$$
\delta r^{2}(t)+D_{0} \int_{0}^{t} d \tau m^{\mathrm{MSD}(\mathrm{MC})}(t-\tau) \delta r^{2}(\tau)=6 D_{0} t
$$

with $D_{0}$ the short-time diffusivity, $\tau_{q}^{\mathrm{s}}=1 /\left(D_{0} q^{2}\right)$, and the initial conditions $\phi_{q}^{\mathrm{s}}(0)=1$ and $\delta r^{2}(0)=0$. This simplification changes the short time transient part of the dynamics, but not its long time properties. In the following, the particle diameter $d$ is used as the unit of length and the unit of time is chosen such that $D_{0}=$ 1/160. Also, since both QA models are found essentially equivalent, only model I will be considered.

For the bulk-like scenario found for small $\phi_{\mathrm{m}}$, it is now well known from extensive studies of one-component bulk systems [14, 41] that, in this case, the essential features of the tagged-particle dynamics are simple reflections of those of the collective dynamics, since it is the latter which actually drives the system through its unique ergodicity breaking transition. So we do not repeat the description of the type B scenario given in Ref. [19], which remains valid in the present case without change, and we omit the analogues for $f_{q}^{\mathrm{s}}$ and $\phi_{q}^{\mathrm{s}}(t)$ of Figs. 5(a), 7(a), and 8 (a) of Ref. [19], in which the evolutions with density of $f_{q}$ and $\phi_{q}(t)$ were reported, since they would display exactly the same patterns. Instead, we concentrate on the distinctive aspects of the single-particle dynamics, i.e., the wave vector dependence of the Lamb-Mössbauer

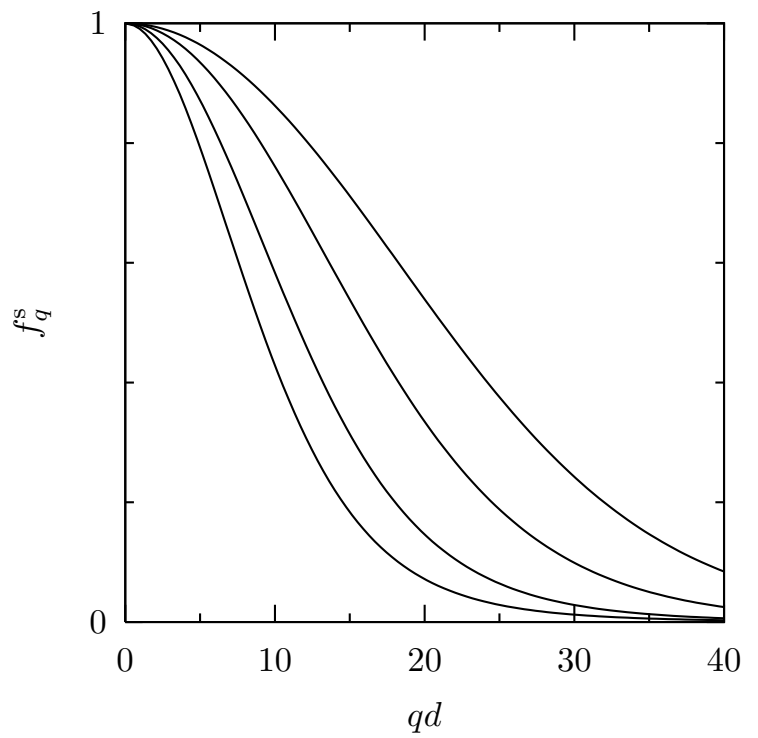

FIG. 4: Wave vector dependence of the single-particle nonergodicity parameter or Lamb-Mössbauer factor $f_{q}^{\mathrm{s}}=$ $\lim _{t \rightarrow \infty} \phi_{q}^{\mathrm{s}}(t)$ for model I at matrix density $\phi_{\mathrm{m}}=0.05$. From left to right, bottom to top: $\phi_{\mathrm{f}}=\phi_{\mathrm{f}}^{\mathrm{c}}, 1.01 \phi_{\mathrm{f}}^{\mathrm{c}}, 1.05 \phi_{\mathrm{f}}^{\mathrm{c}}$, and $1.1 \phi_{\mathrm{f}}^{\mathrm{c}}$.

factor and the time dependence of the mean-squared displacement. As in Ref. [19], we choose $\phi_{\mathrm{m}}=0.05$ for the present illustrations.

Fig. 4 shows $f_{q}^{\text {s }}$ as a function of $q$ at different fluid densities in the glassy phase. As in bulk systems, the curves start at 1 for $q=0$, are approximately Gaussianshaped and widen rapidly when $\phi_{\mathrm{f}}$ is increased. For a given distance to the transition, they are generically narrower than the corresponding curves for the bulk, reflecting the fact that, when $\phi_{\mathrm{m}}$ increases, the system evolves towards a continuous glass transition scenario.

The mean-squared displacements at different fluid densities near the critical one are reported in Fig. 5. For small $t$, short-time diffusion with diffusivity $D_{0}$ is observed. Then, in the ergodic phase, the familiar two-step dynamics typical of the discontinuous ideal glass transition scenario sets in, with, as $\phi_{\mathrm{f}}^{\mathrm{c}}$ is approached, the development of a plateau reflecting transient localization, which crosses over for large $t$ to the diffusion behavior $\delta r^{2}(t)=6 D t$. The long-time diffusion coefficient is generically given by $[41,63]$

$$
D=\frac{D_{0}}{1+D_{0} \int_{0}^{\infty} m^{\mathrm{MSD}(\mathrm{MC})}(t) d t}
$$

and thus vanishes in the present scenario according to the $\alpha$ relaxation scaling law, i.e., $\propto\left|\phi_{\mathrm{f}}-\phi_{\mathrm{f}}^{\mathrm{c}}\right|^{1 / 2 a+1 / 2 b}$, where $a$ and $b$ represent the critical decay and von Schweidler exponents of the collective dynamics, respectively. When the transition density is reached and exceeded, the plateau lasts forever, the tagged particle being permanently trapped. Close to the bifurcation point, the 


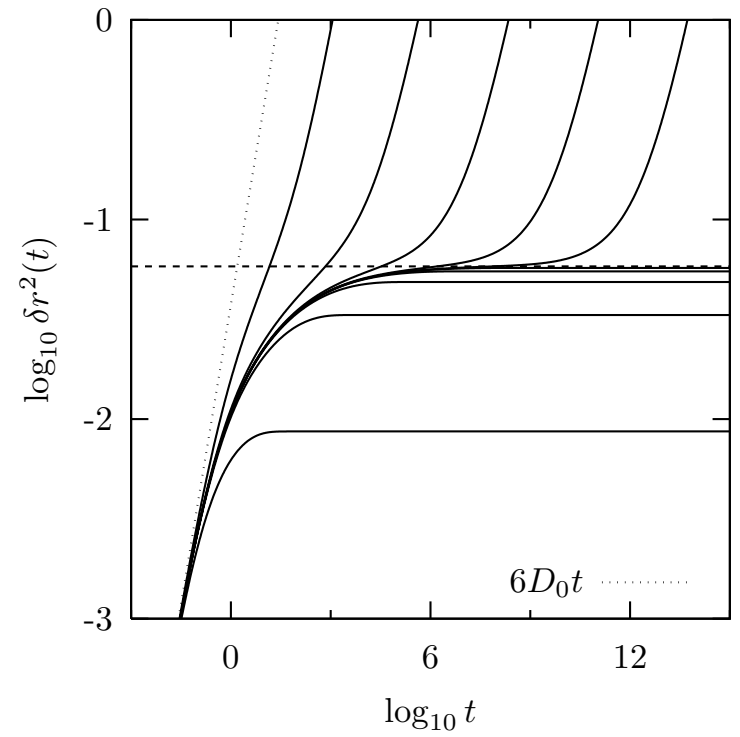

FIG. 5: Time evolution of the mean-squared displacement $\delta r^{2}(t)$ for model I at matrix density $\phi_{\mathrm{m}}=0.05$, in the vicinity of the liquid-glass transition threshold $\phi_{\mathrm{f}}^{\mathrm{c}}$. From left to right, top to bottom: $\phi_{\mathrm{f}}=0.9 \phi_{\mathrm{f}}^{\mathrm{c}}, 0.99 \phi_{\mathrm{f}}^{\mathrm{c}}, 0.999 \phi_{\mathrm{f}}^{\mathrm{c}}, 0.9999 \phi_{\mathrm{f}}^{\mathrm{c}}$, $0.99999 \phi_{\mathrm{f}}^{\mathrm{c}}, 1.00001 \phi_{\mathrm{f}}^{\mathrm{c}}, 1.0001 \phi_{\mathrm{f}}^{\mathrm{c}}, 1.001 \phi_{\mathrm{f}}^{\mathrm{c}}, 1.01 \phi_{\mathrm{f}}^{\mathrm{c}}, 1.1 \phi_{\mathrm{f}}^{\mathrm{c}}$. The horizontal dashed line marks $6 r_{1}^{\mathrm{c} 2}$, with $r_{1}^{\mathrm{c}}$ the critical localization length. The dotted line shows the short-time diffusive behavior $\delta r^{2}(t)=6 D_{0} t$.

localization length $r_{1}$, defined through

$$
\lim _{t \rightarrow \infty} \delta r^{2}(t)=6 r_{1}^{2}
$$

so that

$$
r_{1}^{2}=\left[\int_{0}^{+\infty} \frac{k^{4} d k}{6 \pi^{2}}\left(w_{k}^{(2)} f_{k}^{\mathrm{s}} f_{k}+w_{k}^{(1)} f_{k}^{\mathrm{s}}\right)\right]^{-1},
$$

displays a square-root singularity, $r_{1}-r_{1}^{\mathrm{c}} \propto-\left(\phi_{f}-\phi_{f}^{c}\right)^{1 / 2}$, with $r_{1}^{\mathrm{c}}$ the finite localization length at the transition. In both the diffusive and localized phases, the dynamics in the vicinity of the plateau is described by the same scaling functions as the density fluctuations, with a critical amplitude $\propto\left|\phi_{\mathrm{f}}-\phi_{\mathrm{f}}^{\mathrm{c}}\right|^{1 / 2}$ and a characteristic time scale $\propto\left|\phi_{\mathrm{f}}-\phi_{\mathrm{f}}^{\mathrm{c}}\right|^{-1 / 2 a}$.

The two other scenarios, occuring for moderate and high $\phi_{\mathrm{m}}$, have in common to start with a continuous diffusion-localization transition which affects only the tagged-particle dynamics and leaves the collective quantities free of any singularity. It will now be discussed in some detail and illustrated with computations at $\phi_{\mathrm{m}}=$ 0.1 .

The time evolution of the single-particle density correlation function $\phi_{q}^{\mathrm{s}}(t)$ at $q \simeq 7.09 / d$, corresponding to the main peak of $S_{q}^{\mathrm{ff}(\mathrm{c})}$, is reported in Fig. 6 in a log-log plot (the curves for other values of $q$ are qualitatively similar). In both the diffusive and localized phases near the transition, a single step relaxation is found. When the localization threshold $\phi_{\mathrm{f}}^{1}$ is approached from below, the

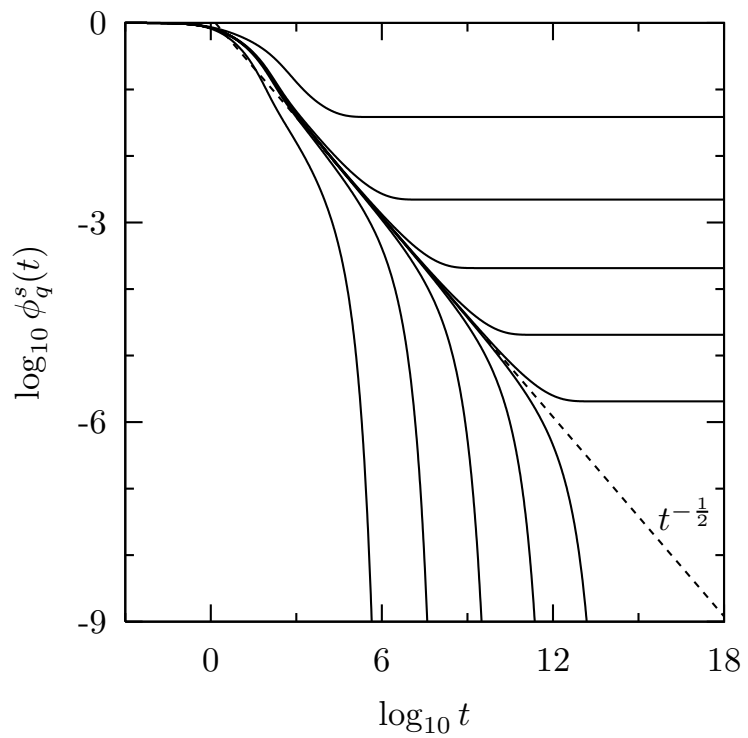

FIG. 6: Time evolution of the single-particle density correlation function $\phi_{q}^{\mathrm{s}}(t)$ at $q \simeq 7.09 / d$ for model $\mathrm{I}$ at matrix density $\phi_{\mathrm{m}}=0.1$, in the vicinity of the localization threshold $\phi_{\mathrm{f}}^{1}$. From left to right, bottom to top: $\phi_{\mathrm{f}}=0.9 \phi_{\mathrm{f}}^{1}, 0.99 \phi_{\mathrm{f}}^{1}$, $0.999 \phi_{\mathrm{f}}^{1}, \quad 0.9999 \phi_{\mathrm{f}}^{1}, 0.99999 \phi_{\mathrm{f}}^{1}, 1.00001 \phi_{\mathrm{f}}^{1}, 1.0001 \phi_{\mathrm{f}}^{1}, 1.001 \phi_{\mathrm{f}}^{1}$, $1.01 \phi_{\mathrm{f}}^{1}, 1.1 \phi_{\mathrm{f}}^{1}$. The dashed line shows the analytically derived critical decay law.

slowing-down of the dynamics manifests itself through the development of a weak long time tail which extends to longer and longer times as the density is increased. At the transition, it lasts indefinitely, and, above the threshold, it progressively recedes and continuously turns into a finite Lamb-Mössbauer factor. From a quantitative point of view, the MCT diffusion-localization transition is most easily understood as a type A bifurcation characterized by an exponent parameter $\lambda=0$ and thus a critical decay exponent $a=1 / 2$. It results that, close to the transition in the localized phase, $f_{q}^{\mathrm{s}} \propto\left(\phi_{\mathrm{f}}-\phi_{\mathrm{f}}^{\mathrm{l}}\right)$. A reduction theorem also holds, stating that, in both phases and for long enough times, the wave vector and time dependence of $\phi_{q}^{\mathrm{s}}(t)$ factorize according to

$$
\phi_{q}^{\mathrm{s}}(t)-\lim _{t \rightarrow \infty} \phi_{q}^{\mathrm{s}}(t)=h_{q}^{\mathrm{s}} G^{\mathrm{s}}(t) .
$$

At the threshold, one gets the critical decay

$$
G^{\mathrm{s}}(t)=\left(t_{0}^{\mathrm{s}} / t\right)^{1 / 2},
$$

where $t_{0}^{\mathrm{s}}$ is a time scale obtained by matching the short and long time dynamics, while, for finite values of $\mid \phi_{\mathrm{f}}-$ $\phi_{\mathrm{f}}^{\mathrm{l}} \mid$, a scaling law is found,

$$
G^{\mathrm{s}}(t)=c^{\mathrm{s}} g^{\mathrm{s}}\left(t / \tau^{\mathrm{s}}\right),
$$

with $c^{\mathrm{s}} \propto\left|\phi_{\mathrm{f}}-\phi_{\mathrm{f}}^{\mathrm{l}}\right|$ and $\tau^{\mathrm{s}} / t_{0}^{\mathrm{s}} \propto\left|\phi_{\mathrm{f}}-\phi_{\mathrm{f}}^{\mathrm{l}}\right|^{-2} \cdot g^{\mathrm{s}}(\hat{t})$ is discussed in detail in Ref. [14] and one finds in particular that

$$
g^{\mathrm{s}}(\hat{t})=\hat{t}^{-1 / 2}
$$




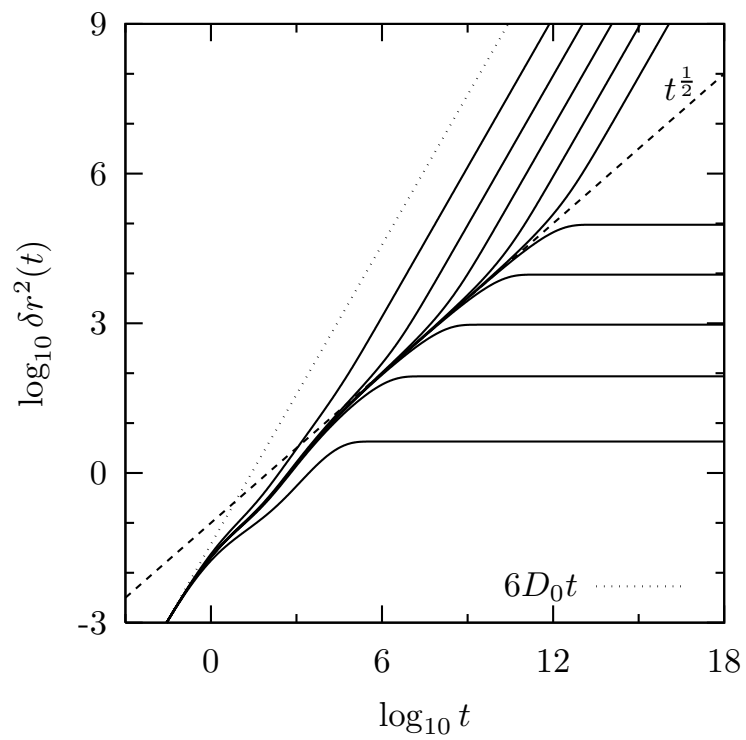

FIG. 7: Time evolution of the mean-squared displacement $\delta r^{2}(t)$ for model I at matrix density $\phi_{\mathrm{m}}=0.1$, in the vicinity of the localization threshold $\phi_{\mathrm{f}}^{\mathrm{l}}$. From left to right, top to bottom: $\phi_{\mathrm{f}}=0.9 \phi_{\mathrm{f}}^{1}, 0.99 \phi_{\mathrm{f}}^{1}, 0.999 \phi_{\mathrm{f}}^{1}, 0.9999 \phi_{\mathrm{f}}^{1}, 0.99999 \phi_{\mathrm{f}}^{1}$, $1.00001 \phi_{\mathrm{f}}^{1}, 1.0001 \phi_{\mathrm{f}}^{\mathrm{l}}, 1.001 \phi_{\mathrm{f}}^{\mathrm{l}}, 1.01 \phi_{\mathrm{f}}^{1}, 1.1 \phi_{\mathrm{f}}^{1}$. The dotted and dashed lines show, respectively, the short-time diffusive behavior $\delta r^{2}(t)=6 D_{0} t$ and the analytically derived critical subdiffusive law $\delta r^{2}(t) \propto t^{1 / 2}$.

for small $\hat{t}$, so that the critical decay law is reached continuously when $\phi_{\mathrm{f}} \rightarrow \phi_{\mathrm{f}}^{1}$. Note that the sign of $\phi_{\mathrm{f}}-\phi_{\mathrm{f}}^{1}$ is irrelevant in the dynamical scaling laws. An important feature of all type A scenarios follows, which is the symmetric departure from the critical decay law (30) at long times for state points located in the ergodic and nonergodic phases at the same distance from the transition. All these analytic predictions are easily confirmed by inspection of Fig. 6 .

Since, according to Eqs. (14)-(16), the time evolution of the mean-squared displacement is controled by that of the single-particle density fluctuations, the anomalous dynamics of the latter near the diffusion-localization transition is naturally transfered to the former. Indeed, as seen in Fig. 7, the long time tail of $\phi_{q}^{\mathrm{s}}(t)$ induces the development in $\delta r^{2}(t)$ of a subdiffusive regime at intermediate times, where $\delta r^{2}(t) \propto t^{1 / 2}$. This regime would persist forever exactly at the transition. For finite $\phi_{\mathrm{f}}-\phi_{\mathrm{f}}^{\mathrm{l}}$, it ends at a time of the order of $\tau^{\mathrm{s}}$, where it crosses over to ordinary diffusion $\delta r^{2}(t)=6 D t$ in the diffusive phase or to a constant $\delta r^{2}(t) \simeq 6 r_{1}^{2}$ in the localized phase. When the transition density is approached from below, the long-time diffusion coefficient vanishes linearly, $D \propto\left|\phi_{\mathrm{f}}-\phi_{\mathrm{f}}^{\mathrm{c}}\right|$, while the localization length diverges according to $r_{\mathrm{l}} \propto\left|\phi_{\mathrm{f}}-\phi_{\mathrm{f}}^{\mathrm{c}}\right|^{-1 / 2}$ when the threshold is approached from above. Here again, all these asymptotic laws can be easily checked in the figure.

Once localization has occured, further increase of the overall density drives the system towards its ideal liquid- glass transition, which can be either discontinuous or continuous. The first case is realized for $\phi_{\mathrm{m}}=0.1$, which was considered above from the point of view of the diffusionlocalization transition. To illustrate the second case, we will choose $\phi_{\mathrm{f}}=0.15$ and use $\phi_{\mathrm{m}}$ as the external control parameter, since, with the QA mixture models studied in this work, it is not possible to find a suitable constant $\phi_{\mathrm{m}}$ trajectory in the dynamical phase diagrams. Note that this exchange of the roles played by $\phi_{\mathrm{f}}$ and $\phi_{\mathrm{m}}$ does not affect the general critical properties of the theory, provided they are formulated in terms of the relevant control parameter. These critical properties will not be discussed in detail. Indeed, it can be very easily demonstrated, by introducing shifted and rescaled tagged-particle density correlation functions so as to eliminate the contributions due to localization, that there is actually no formal difference between the present situation and the simpler case of the bulk-like scenario. So, here as well, the anomalous dynamics of the tagged-particle density fluctuations in the vicinity of the ideal liquid-glass transition is completely controled by the collective dynamics, which imposes the main qualitative and quantitative features of the relaxation.

The complete double transition scenario at $\phi_{\mathrm{m}}=0.1$ is illustrated in Figs. 8, 9, and 10. The data corresponding to the lowest densities provide further illustrations of the features mentioned above for the dynamics near the diffusion-localization transition threshold $\phi_{\mathrm{f}}^{\mathrm{l}}$. This includes the continuous development of the long time tail and finite asymptote in $\phi_{q}^{\mathrm{s}}(t)$, now shown in a semi-log plot in Fig. 8, and the continuous and linear increase of $f_{q}^{\text {s }}$ from zero at $\phi_{\mathrm{f}}^{\mathrm{l}}$, as seen in Fig. 9. Then, approaching the glass transition density $\phi_{\mathrm{f}}^{\mathrm{g}}$, the single-particle density correlation functions start to display a two step decay, which is clearly visible in Fig. 8 and is naturally expected from a type $B$ glass transition scenario. When the second relaxation step disappears because of the spontaneous arrest of the collective dynamics, a discontinuity followed by a square root singularity occurs in the Lamb-Mössbauer factor, as seen in Fig. 9, whose inset shows that $f_{q}^{\text {s }}$ as a function of $q$ always remains bellshaped. All these anomalous behaviors are transmitted to the mean-squared displacement through the memory kernel $m^{\mathrm{MSD}(\mathrm{MC})}(t)$ and result in the two step relaxation pattern visible in Fig. 10.

The analogous data for the double transition scenario at $\phi_{\mathrm{f}}=0.15$ are reported in Figs. 11, 12, and 13. Compared to the previous case, there is no significant difference from the point of view of the dynamics near the diffusion-localization transition threshold $\phi_{\mathrm{m}}^{\mathrm{l}}$. But, as the matrix density is increased, the relaxation of the tagged-particle density fluctuations always remains single stepped, as shown by Fig. 11. The type A liquid-glass transition signals itself by a considerable slowing down of the approach by $\phi_{q}^{\mathrm{s}}(t)$ of its infinite time value, which procedes via an algebraic decay law with the same exponent as the collective dynamics. The single-particle nonergodicity parameter $f_{q}^{\mathrm{s}}$ remains continuous at the transition, 


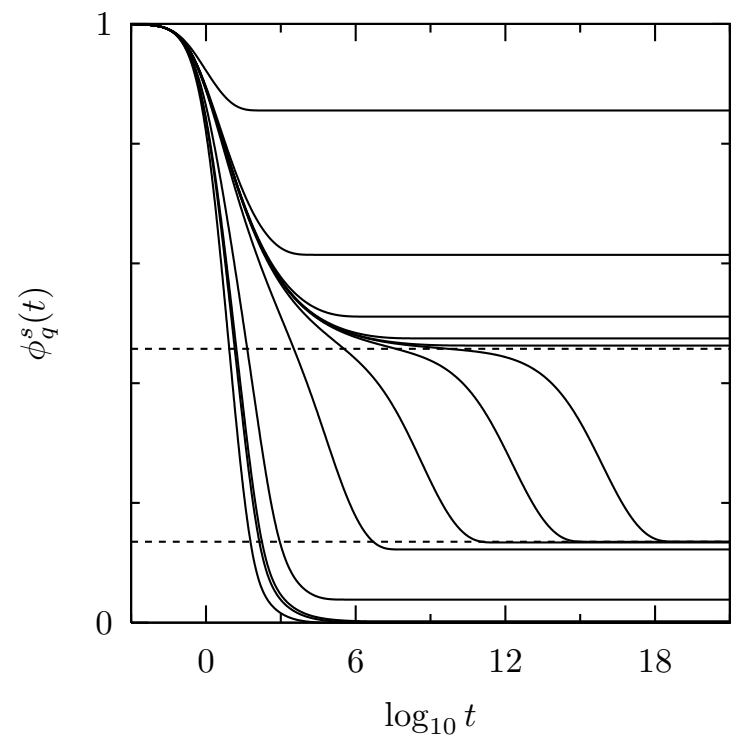

FIG. 8: Time evolution of the single-particle density correlation function $\phi_{q}^{\mathrm{s}}(t)$ at $q \simeq 7.09 / d$ for model I at matrix density $\phi_{\mathrm{m}}=0.1$, in the vicinity of the diffusion-localization and liquid-glass transition thresholds $\phi_{\mathrm{f}}^{1}$ and $\phi_{\mathrm{f}}^{\mathrm{g}}$. From left to right, bottom to top: $\phi_{\mathrm{f}}=0.9 \phi_{\mathrm{f}}^{1}, 0.99 \phi_{\mathrm{f}}^{1}, 1.01 \phi_{\mathrm{f}}^{1}, 1.1 \phi_{\mathrm{f}}^{1} \simeq 0.9 \phi_{\mathrm{f}}^{\mathrm{g}}$, $0.99 \phi_{\mathrm{f}}^{\mathrm{g}}, 0.999 \phi_{\mathrm{f}}^{\mathrm{g}}, 0.9999 \phi_{\mathrm{f}}^{\mathrm{g}}, 0.99999 \phi_{\mathrm{f}}^{\mathrm{g}}, 1.00001 \phi_{\mathrm{f}}^{\mathrm{g}}, 1.0001 \phi_{\mathrm{f}}^{\mathrm{g}}$, $1.001 \phi_{\mathrm{f}}^{\mathrm{g}}, 1.01 \phi_{\mathrm{f}}^{\mathrm{g}}, 1.1 \phi_{\mathrm{f}}^{\mathrm{g}}$. The lower and higher horizontal dashed lines mark the Lamb-Mössbauer factors in the limits $\phi_{\mathrm{f}} \rightarrow \phi_{\mathrm{f}}^{\mathrm{g}-}$ and $\phi_{\mathrm{f}} \rightarrow \phi_{\mathrm{f}}^{\mathrm{g}+}$, respectively.

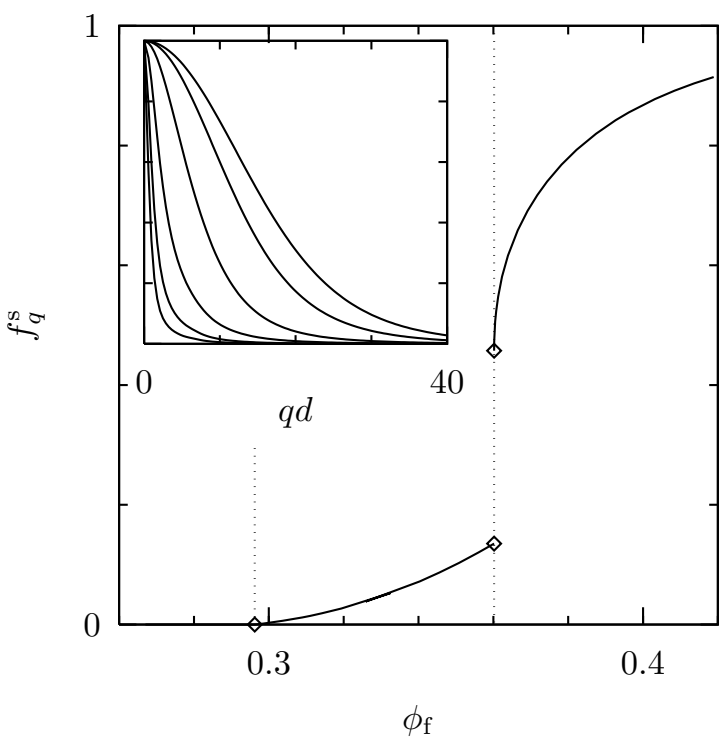

FIG. 9: Density dependence of the single-particle nonergodicity parameter or Lamb-Mössbauer factor $f_{q}^{\mathrm{s}}=\lim _{t \rightarrow \infty} \phi_{q}^{\mathrm{s}}(t)$ at $q \simeq 7.09 / d$ for model I at matrix density $\phi_{\mathrm{m}}=0.1$. Vertical dotted lines and diamonds indicate the diffusion-localization and liquid-glass transitions. Inset: Wave vector dependence of $f_{q}^{\mathrm{s}}$. From left to right, bottom to top: $\phi_{\mathrm{f}}=1.05 \phi_{\mathrm{f}}^{1}, 1.1 \phi_{\mathrm{f}}^{1}$, $\phi_{\mathrm{f}}^{\mathrm{g}-}, \phi_{\mathrm{f}}^{\mathrm{g}+}, 1.05 \phi_{\mathrm{f}}^{\mathrm{g}}$, and $1.1 \phi_{\mathrm{f}}^{\mathrm{g}}$.

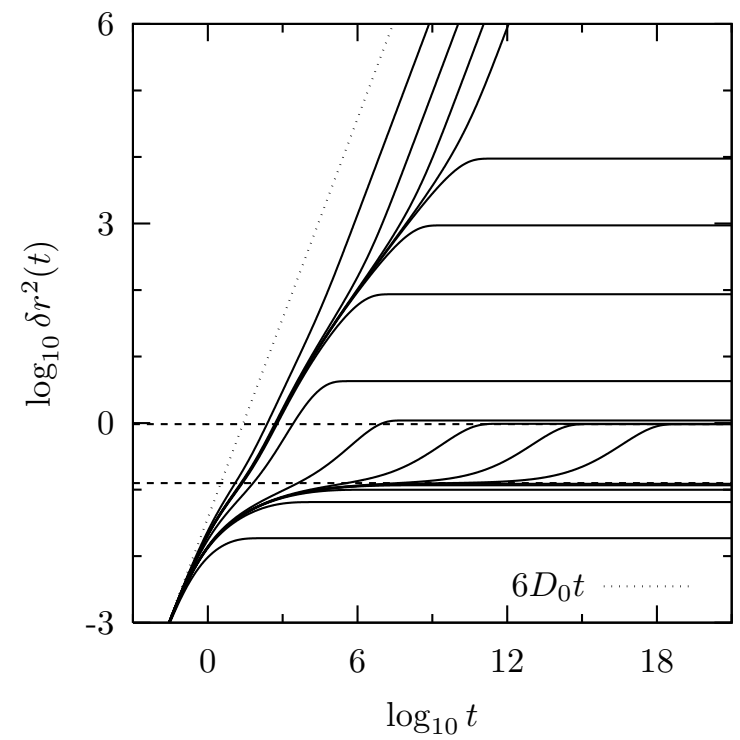

FIG. 10: Time evolution of the mean-squared displacement $\delta r^{2}(t)$ for model I at matrix density $\phi_{\mathrm{m}}=0.1$, in the vicinity of the diffusion-localization and liquid-glass transition thresholds $\phi_{\mathrm{f}}^{1}$ and $\phi_{\mathrm{f}}^{\mathrm{g}}$. From left to right, top to bottom: $\phi_{\mathrm{f}}=0.9 \phi_{\mathrm{f}}^{1}, 0.99 \phi_{\mathrm{f}}^{1}, 0.999 \phi_{\mathrm{f}}^{1}, 0.9999 \phi_{\mathrm{f}}^{1}, 1.0001 \phi_{\mathrm{f}}^{1}, 1.001 \phi_{\mathrm{f}}^{1}$, $1.01 \phi_{\mathrm{f}}^{\mathrm{l}}, 1.1 \phi_{\mathrm{f}}^{1} \simeq 0.9 \phi_{\mathrm{f}}^{\mathrm{g}}, 0.99 \phi_{\mathrm{f}}^{\mathrm{g}}, 0.999 \phi_{\mathrm{f}}^{\mathrm{g}}, 0.9999 \phi_{\mathrm{f}}^{\mathrm{g}}, 0.99999 \phi_{\mathrm{f}}^{\mathrm{g}}$, $1.00001 \phi_{\mathrm{f}}^{\mathrm{g}}, 1.0001 \phi_{\mathrm{f}}^{\mathrm{g}}, 1.001 \phi_{\mathrm{f}}^{\mathrm{g}}, 1.01 \phi_{\mathrm{f}}^{\mathrm{g}}, 1.1 \phi_{\mathrm{f}}^{\mathrm{g}}$. The higher and lower horizontal dashed lines mark $6 r_{1}^{2}$ in the limits $\phi_{\mathrm{f}} \rightarrow \phi_{\mathrm{f}}^{\mathrm{g}-}$ and $\phi_{\mathrm{f}} \rightarrow \phi_{\mathrm{f}}^{\mathrm{g}+}$, respectively. The dotted line shows the shorttime diffusive behavior $\delta r^{2}(t)=6 D_{0} t$.

which is only marked by an abrupt but finite change of slope visible in Fig. 12. Finally, here as well, the time evolution of the mean-squared displacement $\delta r^{2}(t)$, reported in Fig. 13, is directly influenced by this anomalous relaxation of the density fluctuations and, as a result, shows a regime of continuous critical dynamics in the localized phase near the glass transition point.

Like in Ref. [19], we shall leave aside the peculiar dynamics found in the vicinity of the state points corresponding to higher-order singularities or crossing points, either fully embedded in the liquid-glass transition lines (points $\mathrm{E}$ and $\mathrm{C}$ ) or at their junctions with the diffusionlocalization transition lines (points $\mathrm{T}$ ). They certainly are interesting, but such a study would be quite technical, requiring fine-tuning of the parameters of the models and refined mathematical tools $[14,64,65,66,67]$. So we prefer to rather concentrate on the generic scenarios, which are the most likely to be observed in experiments or computer simulations, and we keep such an advanced discussion for future work, when it will be useful, for instance, if relevant data become available.

\section{DISCUSSION AND CONCLUSION}

In this paper, a MCT for the slow dynamics of a tagged particle moving in a fluid adsorbed in a disordered porous 


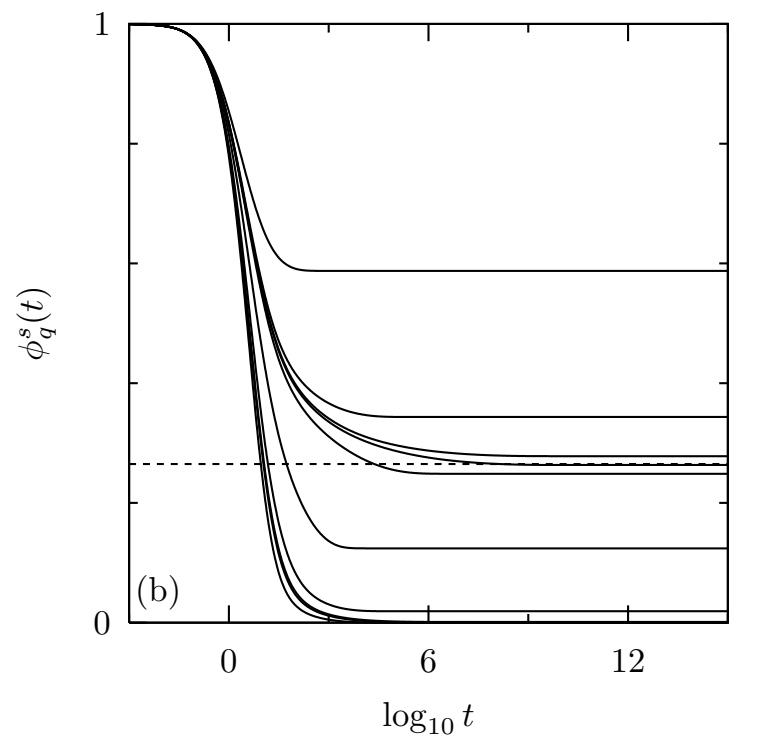

FIG. 11: Time evolution of the single-particle density correlation function $\phi_{q}^{\mathrm{s}}(t)$ at $q \simeq 7.09 / d$ for model $\mathrm{I}$ at fluid density $\phi_{\mathrm{f}}=0.15$, in the vicinity of the diffusion-localization and liquid-glass transition thresholds $\phi_{\mathrm{m}}^{1}$ and $\phi_{\mathrm{m}}^{\mathrm{g}}$. From left to right, bottom to top: $\phi_{\mathrm{m}}=0.9 \phi_{\mathrm{m}}^{1}, 0.99 \phi_{\mathrm{m}}^{1}, 1.01 \phi_{\mathrm{m}}^{1}, 1.1 \phi_{\mathrm{m}}^{1}$, $0.9 \phi_{\mathrm{m}}^{\mathrm{g}}, 0.99 \phi_{\mathrm{m}}^{\mathrm{g}}, 0.999 \phi_{\mathrm{m}}^{\mathrm{g}}, 1.001 \phi_{\mathrm{m}}^{\mathrm{g}}, 1.01 \phi_{\mathrm{m}}^{\mathrm{g}}, 1.1 \phi_{\mathrm{m}}^{\mathrm{g}}$. The horizontal dashed line marks the Lamb-Mössbauer factor at $\phi_{\mathrm{m}}^{\mathrm{g}}$.

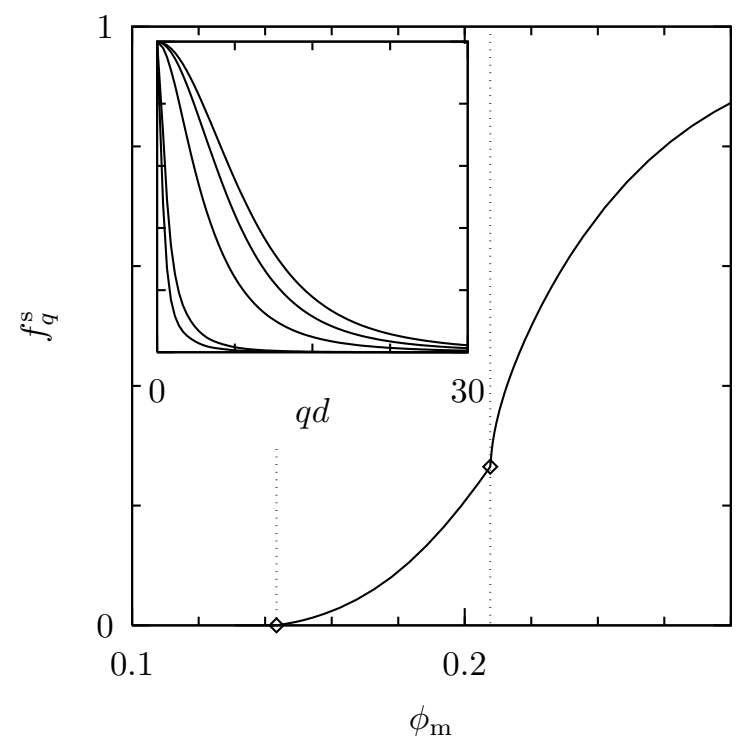

FIG. 12: Density dependence of the single-particle nonergodicity parameter or Lamb-Mössbauer factor $f_{q}^{\mathrm{s}}=\lim _{t \rightarrow \infty} \phi_{q}^{\mathrm{s}}(t)$ at $q \simeq 7.09 / d$ for model I at fluid density $\phi_{\mathrm{f}}=0.15$. Vertical dotted lines and diamonds indicate the diffusion-localization and liquid-glass transitions. Inset: Wave vector dependence of $f_{q}^{\mathrm{s}}$. From left to right, bottom to top: $\phi_{\mathrm{m}}=1.05 \phi_{\mathrm{m}}^{1}, 1.1 \phi_{\mathrm{m}}^{1}$, $\phi_{\mathrm{m}}^{\mathrm{g}}, 1.05 \phi_{\mathrm{m}}^{\mathrm{g}}$, and $1.1 \phi_{\mathrm{m}}^{\mathrm{g}}$.

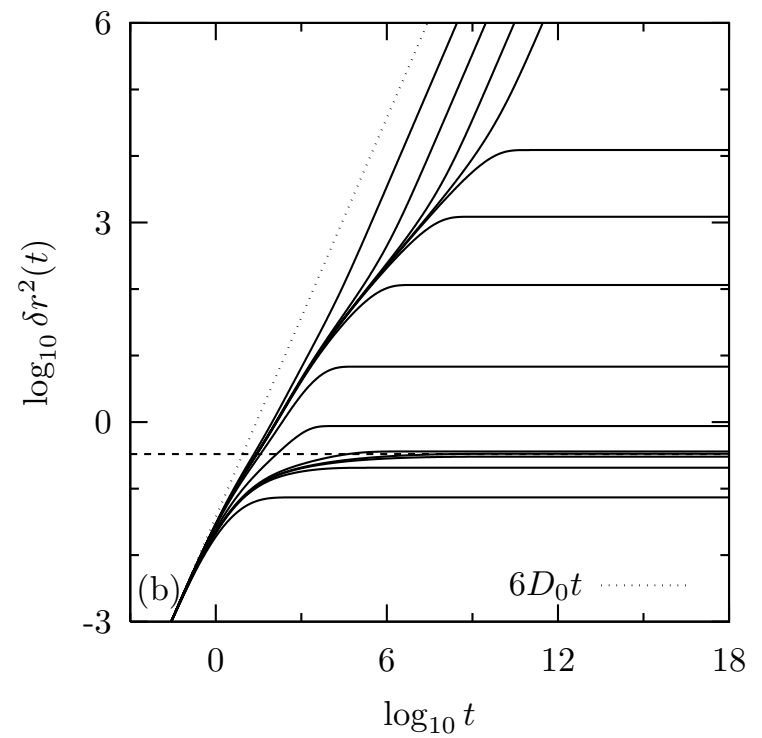

FIG. 13: Time evolution of the mean-squared displacement $\delta r^{2}(t)$ for model I at fluid density $\phi_{\mathrm{f}}=0.15$, in the vicinity of the diffusion-localization and liquid-glass transition thresholds $\phi_{\mathrm{m}}^{\mathrm{l}}$ and $\phi_{\mathrm{m}}^{\mathrm{g}}$. From left to right, top to bottom: $\phi_{\mathrm{m}}=0.9 \phi_{\mathrm{m}}^{1}, 0.99 \phi_{\mathrm{m}}^{1}, 0.999 \phi_{\mathrm{m}}^{1}, 0.9999 \phi_{\mathrm{m}}^{1}, 1.0001 \phi_{\mathrm{m}}^{1}, 1.001 \phi_{\mathrm{m}}^{1}$, $1.01 \phi_{\mathrm{m}}^{1}, 1.1 \phi_{\mathrm{m}}^{1}, 0.9 \phi_{\mathrm{m}}^{\mathrm{g}}, 0.99 \phi_{\mathrm{m}}^{\mathrm{g}}, 0.999 \phi_{\mathrm{m}}^{\mathrm{g}}, 1.001 \phi_{\mathrm{m}}^{\mathrm{g}}, 1.01 \phi_{\mathrm{m}}^{\mathrm{g}}$, $1.1 \phi_{\mathrm{m}}^{\mathrm{g}}$. The horizontal dashed line marks $6 r_{1}^{2}$ at $\phi_{\mathrm{m}}^{\mathrm{g}}$. The dotted line shows the short-time diffusive behavior $\delta r^{2}(t)=6 D_{0} t$.

solid has been developed. It complements previous work on the collective dynamics reported in Refs. [17, 18, 19], so that one might now consider as complete the modecoupling theoretical framework for the study of the dynamics of density fluctuations in QA systems.

From a formal point of view, the newly derived equations keep the features of those of Ref. [19] which were found appealing. They are universality, in the sense that the eventual dynamical equations do not contain any explicit reference to the precise nature of the random environment in which the fluid evolves, and a very close similarity with other mode-coupling equations previously derived in comparable contexts. So, the overall structure of the theory appears quite satisfactory. Note however that this turns out not to be a completely trivial task to obtain such equations which display these features and also avoid certain subtle inconsistencies. Indeed, as shown in Sec. III, special care seems to be generically needed in order to properly handle the peculiar correlations in QA systems, for reasons which are not fully clear at the moment. In view of these difficulties, it is hoped that the present approach can be useful in the future as a guide for developments based on other formalisms.

From a physical point of view, the main result of the present work is the prediction of a continuous diffusionlocalization transition which can occur before and independently of the liquid-glass transition in situations of strong confinement. It is characterized by the emergence of various dynamical anomalies, in particular, by the de- 
velopment of an algebraic long time tail in the taggedparticle density correlators and by the opening of a subdiffusive regime in the mean-squared displacement.

Dealing with the problem of a tagged particle moving in a random medium, the possibility of a diffusionlocalization transition is a natural expectation. However, the prediction that the single-particle dynamics can become singular and nonergodic while the collective dynamics remains regular and ergodic might look suspect, owing to the fact that the former contributes to the latter, of which it represents the so-called self part. In fact, the difficulty is only superficial. Firstly, it should be remembered that the theory for the collective dynamics and the definition of the ideal liquid-glass transition are formulated in terms of the connected fluid density correlation functions, while the single-particle density correlation function is the self part of the total fluid density correlation function. Because the random external field provided by the fixed matrix induces nonzero average density fluctuations in the fluid at equilibrium, the latter, defined as

$$
\phi_{q}^{\mathrm{T}}(t)=\frac{\overline{\left\langle\rho_{\mathbf{q}}^{\mathrm{f}}(t) \rho_{-\mathbf{q}}^{\mathrm{f}}(0)\right\rangle}}{N_{\mathrm{f}} S_{q}^{\mathrm{ff}}}
$$

with $S_{q}^{\mathrm{ff}}=\overline{\left\langle\rho_{\mathbf{q}}^{\mathrm{f}} \rho_{-\mathbf{q}}^{\mathrm{f}}\right\rangle} / N_{\mathrm{f}}=S_{q}^{\mathrm{ff}(\mathrm{c})}+S_{q}^{\mathrm{ff}(\mathrm{b})}$, can be written as

$$
\phi_{q}^{\mathrm{T}}(t)=\frac{S_{q}^{\mathrm{ff}(\mathrm{c})}}{S_{q}^{\mathrm{ff}}} \phi_{q}(t)+\frac{S_{q}^{\mathrm{ff}(\mathrm{b})}}{S_{q}^{\mathrm{ff}}}
$$

from which it is clear that $\phi_{q}^{\mathrm{T}}(t)$ never decays to zero in a QA system. So, one might say that the fluid density fluctuations are never really ergodic in a QA mixture, in the loose sense that they saturate at a finite value, like in a bulk glassy system. Secondly, based on the results of the MCT for one-component bulk systems [14] and on the prevalence of rudimentary approximations like Vineyard's [68], it is often believed that the collective and self density fluctuations should generically behave more or less the same way. This is not correct. In fact, one can easily demonstrate, using the memory function formalism, that the total and single-particle density correlation functions are completely independent, formally decoupled objects [69], so that the general case is rather the contrary, i.e., the two functions display quite different behaviors [70]. Thus, despite appearances, the pure diffusion-localization transition scenario predicted in this work, which is analogous to the one predicted years ago for bulk binary mixtures with large size asymmetry $[22,23,24]$, is not marred by any inconsistency.

An interesting aspect of this prediction, that we expect to be quite general, is that it demonstrates the possibility, when strong static fluid correlations [encoded here in $h^{\mathrm{ss}(\mathrm{b})}(r)$ ] are present in confinement, that the single-particle dynamics develops slow relaxational features which have no relation whatsoever with glassy dynamics, if the latter is to be understood in the usual way as a collective dynamical phenomenon. We believe that this finding could be important for the design and the interpretation of experiments and computer simulations on the dynamics of confined glassforming systems. Indeed, the idea that confinement can be responsible for the appearence of additional slow dynamical processes which blend with the glassy dynamics and might obscure its features is definitely not novel [7, 8] (see Refs. [71, 72] for a specific experimental observation of such a process). But it does not seem to have been realized previously that there could be such a major difference in the way some of these processes are reflected in collective and singleparticle quantities, being essentially invisible in the former and possibly dominant in the latter. This suggests that, if one is mainly interested in understanding the effect of confinement on the glass transition, it should be easier to concentrate on collective dynamical quantities, and that, if single-particle data are to be considered, one should be prepared to face more complex dynamical scenarios which might result from the interplay between the glassy dynamics and confinement-induced single-particle relaxation processes.

Finally, all the predictions made in the present and previous papers $[17,18,19]$ can in principle be tested by computer simulations, in order to judge the quality of the proposed theory. As far as the single-particle dynamics is concerned, this should not be too challenging, since, in the past few years, a significant number of simulation studies have actually been already reported, which precisely dealt with the tagged-particle dynamics in QA systems $[33,53,73,74,75,76,77,78,79,80,81]$. Unfortunately, in all these studies, either the model was simple, but the focus was not on slow dynamics, or the glassy dynamics was explored, but the fluid model was quite complex, so that direct comparisons with the results of the present work are never possible. We hope that the situation can improve very soon.

\section{APPENDIX A: REPLICA ORNSTEIN-ZERNIKE EQUATIONS}

For reference, we quote in this appendix the replica Ornstein-Zernike equations relating the various pair correlation functions mentioned in the main text.

For the fluid-matrix part, they read [28, 29, 30, 31]

$$
\begin{gathered}
\hat{h}_{q}^{\mathrm{mm}}=\hat{c}_{q}^{\mathrm{mm}}+n_{\mathrm{m}} \hat{c}_{q}^{\mathrm{mm}} \hat{h}_{q}^{\mathrm{mm}}, \\
\hat{h}_{q}^{\mathrm{fm}}=\hat{c}_{q}^{\mathrm{fm}}+n_{\mathrm{m}} \hat{c}_{q}^{\mathrm{fm}} \hat{h}_{q}^{\mathrm{mm}}+n_{\mathrm{f}} \hat{c}_{q}^{\mathrm{ff}(\mathrm{c})} \hat{h}_{q}^{\mathrm{fm}}, \\
\hat{h}_{q}^{\mathrm{ff}(\mathrm{b})}=\hat{c}_{q}^{\mathrm{ff}(\mathrm{b})}+n_{\mathrm{m}} \hat{c}_{q}^{\mathrm{fm}} \hat{h}_{q}^{\mathrm{fm}}+n_{\mathrm{f}} \hat{c}_{q}^{\mathrm{ff}(\mathrm{c})} \hat{h}_{q}^{\mathrm{ff}(\mathrm{b})}+n_{\mathrm{f}} \hat{c}_{q}^{\mathrm{ff}(\mathrm{b})} \hat{h}_{q}^{\mathrm{ff}(\mathrm{c})}, \\
\hat{h}_{q}^{\mathrm{ff}(\mathrm{c})}=\hat{c}_{q}^{\mathrm{ff}(\mathrm{c})}+n_{\mathrm{f}} \hat{c}_{q}^{\mathrm{ff}(\mathrm{c})} \hat{h}_{q}^{\mathrm{ff}(\mathrm{c})},
\end{gathered}
$$

with $\hat{c}_{q}^{\mathrm{ff}}=\hat{c}_{q}^{\mathrm{ff}(\mathrm{c})}+\hat{c}_{q}^{\mathrm{ff}(\mathrm{b})}$ and $\hat{h}_{q}^{\mathrm{ff}}=\hat{h}_{q}^{\mathrm{ff}(\mathrm{c})}+\hat{h}_{q}^{\mathrm{ff}(\mathrm{b})} \cdot$ As usual, $h$ and $c$ denote total and direct correlation functions, respectively. $\hat{f}$ denotes the Fourier transform of $f$ and 
the superscripts have the same meaning as in the main text.

They can be easily combined with the definitions of the structure factors,

$$
\begin{gathered}
S_{q}^{\mathrm{mm}}=1+n_{\mathrm{m}} \hat{h}_{q}^{\mathrm{mm}}, \\
S_{q}^{\mathrm{fm}}=\sqrt{n_{\mathrm{f}} n_{\mathrm{m}}} \hat{h}_{q}^{\mathrm{fm}}, \\
S_{q}^{\mathrm{ff}(\mathrm{c})}=1+n_{\mathrm{f}} \hat{h}_{q}^{\mathrm{ff}(\mathrm{c})}, \\
S_{q}^{\mathrm{ff(}(\mathrm{b})}=n_{\mathrm{f}} \hat{h}_{q}^{\mathrm{ff}(\mathrm{b})},
\end{gathered}
$$

(remember that $S_{q}^{\mathrm{ff}}=S_{q}^{\mathrm{ff}(\mathrm{c})}+S_{q}^{\mathrm{ff}(\mathrm{b})}$, hence $S_{q}^{\mathrm{ff}}=1+$ $\left.n_{\mathrm{f}} \hat{h}_{q}^{\mathrm{ff}}\right)$ to express them in terms of the direct correlation functions (see Appendix A of Ref. [19]).

For the single-particle part, the relevant equations, which are limiting cases of those reported in Ref. [32], read

$$
\begin{aligned}
& \hat{h}_{q}^{\mathrm{sm}}=\hat{c}_{q}^{\mathrm{sm}}+n_{\mathrm{m}} \hat{c}_{q}^{\mathrm{sm}} \hat{h}_{q}^{\mathrm{mm}}+n_{\mathrm{f}} \hat{c}_{q}^{\mathrm{sf}(\mathrm{c})} \hat{h}_{q}^{\mathrm{fm}}, \\
& \hat{h}_{q}^{\mathrm{sf}(\mathrm{b})}=\hat{c}_{q}^{\mathrm{sf}(\mathrm{b})}+n_{\mathrm{m}} \hat{c}_{q}^{\mathrm{sm}} \hat{h}_{q}^{\mathrm{fm}}+n_{\mathrm{f}} \hat{\mathrm{c}}_{q}^{\mathrm{sf}(\mathrm{b})} \hat{h}_{q}^{\mathrm{ff}(\mathrm{c})}+n_{\mathrm{f}} \hat{\mathrm{c}}_{q}^{\mathrm{ff}(\mathrm{c})} \hat{h}_{q}^{\mathrm{ff}(\mathrm{b})}, \\
& \hat{h}_{q}^{\mathrm{sf}(\mathrm{c})}=\hat{c}_{q}^{\mathrm{sf}(\mathrm{c})}+n_{\mathrm{f}} \hat{c}_{q}^{\mathrm{ff}(\mathrm{c})} \hat{h}_{q}^{\mathrm{ff}(\mathrm{c})}, \\
& \hat{h}_{q}^{\mathrm{ss}(\mathrm{b})}=\hat{c}_{q}^{\mathrm{ss}(\mathrm{b})}+n_{\mathrm{m}} \hat{c}_{q}^{\mathrm{sm}} \hat{h}_{q}^{\mathrm{sm}}+n_{\mathrm{f}} \hat{c}_{q}^{\mathrm{sf}(\mathrm{c})} \hat{h}_{q}^{\mathrm{sf}(\mathrm{b})}+n_{\mathrm{f}} \hat{c}_{q}^{\mathrm{sf}(\mathrm{b})} \hat{h}_{q}^{\mathrm{sf}(\mathrm{c})}, \\
& \hat{h}_{q}^{\mathrm{ss}(\mathrm{c})}=\hat{c}_{q}^{\mathrm{ss}(\mathrm{c})}+n_{\mathrm{f}} \hat{c}_{q}^{\mathrm{sf}(\mathrm{c})} \hat{h}_{q}^{\mathrm{sf}(\mathrm{c})},
\end{aligned}
$$

with $\hat{c}_{q}^{\mathrm{sf}}=\hat{c}_{q}^{\mathrm{sf}(\mathrm{c})}+\hat{c}_{q}^{\mathrm{sf}(\mathrm{b})}, \hat{h}_{q}^{\mathrm{sf}}=\hat{h}_{q}^{\mathrm{sf}(\mathrm{c})}+\hat{h}_{q}^{\mathrm{sf}(\mathrm{b})}, \hat{c}_{q}^{\mathrm{ss}}=\hat{c}_{q}^{\mathrm{ss}(\mathrm{c})}+$ $\hat{c}_{q}^{\mathrm{ss}(\mathrm{b})}$, and $\hat{h}_{q}^{\mathrm{ss}}=\hat{h}_{q}^{\mathrm{ss}(\mathrm{c})}+\hat{h}_{q}^{\mathrm{ss}(\mathrm{b})}$.

\section{APPENDIX B: MODE-COUPLING EQUATIONS FOR A MIXTURE ADSORBED IN A DISORDERED POROUS SOLID}

In this appendix, we report the MCT equations for the dynamics of a mixture adsorbed in a disordered porous matrix. They are derived following an obvious generalization of the procedure outlined in appendix B of Ref. [19] and are thus given without proof.

The motivation for this appendix is twofold. First, these equations are needed in order to derive those for the tagged-particle dynamics, which are the main result of the present work. Second, most simple glassformer models which are considered in the literature for comparisons between theoretical predictions and intensive computer simulation results are binary mixtures, for the reason that they are much less prone to crystallisation than one-component systems. So it is likely that sooner or later a version of the theory dealing with adsorbed mixtures will become useful, for instance, in order to perform quantitative tests like the ones reported in Refs. [82, 83] for bulk systems.

We consider a quenched-annealed multicomponent mixture containing $n$ fluid components. Each of them consists of $N_{\alpha}$ particles of mass $m_{\alpha}(1 \leq \alpha \leq n)$. The total fluid particle number is $N_{\mathrm{f}}=\sum_{\alpha=1}^{n} N_{\alpha}$ and the fluid number fractions are defined as $x_{\alpha}=N_{\alpha} / N_{\mathrm{f}}$. The system is at temperature $T$ and has volume $V$, hence the total fluid density is $n_{\mathrm{f}}=N_{\mathrm{f}} / V$. Note that it is not necessary to provide any information on the disordered matrix, since only quantities characterizing the fluid component of the QA system will appear in the final results.

The dynamical variables of interest are the timedependent density fluctuations of the different fluid species,

$$
\rho_{\mathbf{q}}^{\alpha}(t)=\sum_{j=1}^{N_{\alpha}} e^{i \mathbf{q} \mathbf{r}_{j}^{\alpha}(t)},
$$

where $\mathbf{r}_{j}^{\alpha}(t)$ is the position of the fluid particle $j$ of type $\alpha$ at time $t$. From these quantities, one can form the relevant static structure factors, connected,

$$
S_{q}^{\alpha \beta(\mathrm{c})}=\frac{1}{N_{\mathrm{f}}} \overline{\left\langle\delta \rho_{\mathbf{q}}^{\alpha} \delta \rho_{-\mathbf{q}}^{\beta}\right\rangle},
$$

with $\delta \rho_{\mathbf{q}}^{\alpha}(t)=\rho_{\mathbf{q}}^{\alpha}(t)-\left\langle\rho_{\mathbf{q}}^{\alpha}\right\rangle$, and blocked or disconnected,

$$
S_{q}^{\alpha \beta(\mathrm{b})}=\frac{1}{N_{\mathrm{f}}} \overline{\left\langle\rho_{\mathbf{q}}^{\alpha}\right\rangle\left\langle\rho_{-\mathbf{q}}^{\beta}\right\rangle},
$$

as well as the nonnormalized time-dependent connected density fluctuation autocorrelation functions

$$
F_{q}^{\alpha \beta}(t)=\frac{1}{N_{\mathrm{f}}} \overline{\left\langle\delta \rho_{\mathbf{q}}^{\alpha}(t) \delta \rho_{-\mathbf{q}}^{\beta}(0)\right\rangle} .
$$

These functions obey standard generalized Langevin equations which read in matrix form,

$$
\ddot{\mathbf{F}}_{q}(t)+\boldsymbol{\Omega}_{q}^{2} \mathbf{F}_{q}(t)+\int_{0}^{t} d \tau \mathbf{M}_{q}(t-\tau) \dot{\mathbf{F}}_{q}(\tau)=\mathbf{0},
$$

with initial conditions $\mathbf{F}_{q}(0)=\mathbf{S}_{q}^{(\mathrm{c})}$ and $\dot{\mathbf{F}}_{q}(0)=\mathbf{0}$, and a frequency matrix given by

$$
\left[\boldsymbol{\Omega}_{q}^{2}\right]^{\alpha \beta}=\frac{q^{2} k_{B} T x_{\alpha}}{m_{\alpha}}\left[\mathbf{S}_{q}^{(\mathrm{c})-1}\right]^{\alpha \beta},
$$

where $\mathbf{S}_{q}^{(\mathrm{c})-1}$ is the matrix inverse of $\mathbf{S}_{q}^{(\mathrm{c})}$.

The MCT provides an expression for the kernel $\mathbf{M}_{q}(t)$, which reads $\mathbf{M}_{q}(t)=\boldsymbol{\Gamma}_{q} \delta(t)+\mathbf{M}_{q}^{(\mathrm{MC})}(t)$, where $\boldsymbol{\Gamma}_{q}$ is a matrix of friction coefficients associated with fast dynamical processes and

$$
M_{q}^{\alpha \alpha^{\prime}(\mathrm{MC})}(t)=\frac{q^{2} k_{B} T}{m_{\alpha} x_{\alpha^{\prime}}}\left[m_{q}^{\alpha \alpha^{\prime}(2)}(t)+m_{q}^{\alpha \alpha^{\prime}(1)}(t)\right],
$$

with

$m_{q}^{\alpha \alpha^{\prime}(2)}(t)=\frac{1}{2} n_{\mathrm{f}} \int \frac{d^{3} \mathbf{k}}{(2 \pi)^{3}} \sum_{\beta, \gamma, \beta^{\prime}, \gamma^{\prime}=1}^{n} V_{\mathbf{q}, \mathbf{k}}^{\alpha \beta(2)} V_{\mathbf{q}, \mathbf{k}}^{\alpha^{\prime} \beta^{\prime} \gamma^{\prime}(2)} F_{k}^{\beta \beta^{\prime}}(t) F_{\mid \mathbf{q}-\mathbf{k}}^{\gamma \gamma^{\prime}}$ 
and

with the generalized diffusion coefficient $\tilde{D}_{q}(z)$ given by

$m_{q}^{\alpha \alpha^{\prime}(1)}(t)=n_{\mathrm{f}} \int \frac{d^{3} \mathbf{k}}{(2 \pi)^{3}} \sum_{\beta, \beta^{\prime}=1}^{n} V_{\mathbf{q}, \mathbf{k}}^{\alpha \beta(1)} V_{\mathbf{q}, \mathbf{k}}^{\alpha^{\prime} \beta^{\prime}(1)} F_{k}^{\beta \beta^{\prime}}(t) S_{|\mathbf{q}-\mathbf{k}|}^{\alpha \alpha^{\prime}(\mathrm{b})}$

$$
\tilde{D}_{q}(z)=\frac{-k_{B} T / m_{\mathrm{s}}}{z+\omega_{q}^{2} \tilde{m}_{q}^{\mathrm{s}}(z)} .
$$

The vertices are given by

$$
\begin{aligned}
V_{\mathbf{q}, \mathbf{k}}^{\alpha \beta \gamma(2)} & =\frac{\mathbf{q} \cdot \mathbf{k}}{q^{2}} \delta_{\alpha \gamma} \hat{c}_{k}^{\alpha \beta(\mathrm{c})}+\frac{\mathbf{q} \cdot(\mathbf{q}-\mathbf{k})}{q^{2}} \delta_{\alpha \beta} \hat{c}_{|\mathbf{q}-\mathbf{k}|}^{\alpha \gamma(\mathrm{c})}, \\
V_{\mathbf{q}, \mathbf{k}}^{\alpha \beta(1)} & =\frac{\mathbf{q} \cdot \mathbf{k}}{q^{2}} \hat{c}_{k}^{\alpha \beta(\mathrm{c})}+\frac{\mathbf{q} \cdot(\mathbf{q}-\mathbf{k})}{q^{2}} \frac{1}{n_{\mathrm{f}} x_{\alpha}} \delta_{\alpha \beta},
\end{aligned}
$$

where the $\hat{c}_{q}^{\alpha \beta(\mathrm{c})}$ 's are the Fourier transforms of the connected direct correlation functions [32].

The equations for the tagged-particle dynamics are obtained by considering a species $\sigma$ in the limit of a vanishing number fraction. One then has $\phi_{q}^{\mathrm{s}}(t)=$ $\lim _{x_{\sigma} \rightarrow 0} F_{q}^{\sigma \sigma}(t) / x_{\sigma}$. After tedious but straightforward algebra, one finds the usual Langevin equation (11) and

$$
m_{q}^{\mathrm{s}(\mathrm{MC})}(t)=m_{q}^{\mathrm{s}(2)}(t)+m_{q}^{\mathrm{s}(1)}(t)
$$

with

$m_{q}^{\mathrm{s}(2)}(t)=n_{\mathrm{f}} \int \frac{d^{3} \mathbf{k}}{(2 \pi)^{3}} \sum_{\beta, \beta^{\prime}=1}^{n}\left[\frac{\mathbf{q} \cdot(\mathbf{q}-\mathbf{k})}{q^{2}}\right]^{2} \hat{c}_{|\mathbf{q}-\mathbf{k}|}^{\mathrm{s} \beta(\mathrm{c})} \hat{c}_{|\mathbf{q}-\mathbf{k}|}^{\mathrm{s} \beta^{\prime}(\mathrm{c})} F_{\mid \mathbf{q}-\mathbf{k}}^{\beta \beta^{\prime}}$

and

$$
m_{q}^{\mathrm{s}(1)}(t)=\int \frac{d^{3} \mathbf{k}}{(2 \pi)^{3}}\left[\frac{\mathbf{q} \cdot(\mathbf{q}-\mathbf{k})}{q^{2}}\right]^{2} h_{|\mathbf{q}-\mathbf{k}|}^{\mathrm{ss}(\mathrm{b})} \phi_{k}^{\mathrm{s}}(t) .
$$

The $\hat{c}_{q}^{\mathrm{s} \alpha(\mathrm{c})}$ 's and $\hat{h}_{q}^{\mathrm{ss}(\mathrm{b})}$ are the Fourier transforms of the connected single-particle-fluid direct correlation functions and of the single-particle-single-particle blocked total correlation function, respectively [32].

The equations reported in the main body of the paper are immediately obtained for $n=1$.

\section{APPENDIX C: HYDRODYNAMIC APPROXIMATION FOR THE DIFFUSION-LOCALIZATION TRANSITION}

In this appendix, the results of a generalized hydrodynamic approximation, in which the wave vector dependence of the MCT equations is simplified, are reported for the dynamics in the vicinity of the diffusion-localization transition. They represent an extension of Leutheusser's theory [26] to finite fluid densities.

The theory is best obtained by considering Laplace transformed dynamical quantities, defined as $\tilde{\phi}(z)=$ $i \int_{0}^{\infty} d t e^{i z t} \phi(t)$ for $\operatorname{Im} z>0$. Equation (11) then becomes

$$
\tilde{\phi}_{q}^{\mathrm{s}}(z)=\frac{-1}{z+q^{2} \tilde{D}_{q}(z)}
$$

The hydrodynamic approximation simply amounts to neglecting the $q$ dependence of $\tilde{D}_{q}(z)$ in Eq. $(\mathrm{C} 1)$, where it is replaced by its $q \rightarrow 0$ limit

$$
\tilde{D}(z)=\frac{-k_{B} T / m_{\mathrm{s}}}{z+\tilde{m}^{\mathrm{MSD}}(z) k_{B} T / m_{\mathrm{s}}} .
$$

Further progress is made by noting that it is not necessary to give a detailed account of the bilinear mode-coupling term in $m^{\mathrm{MSD}}(t)$ if one is interested in the anomalous dynamics near the diffusion-localization threshold. Indeed, on the one hand, it is clear that this term can be singular when $n_{\mathrm{f}}$ is very small, because the diffusion-localization and liquid-glass transition lines are then very close, but it is also very small, because of the explicit factor $n_{\mathrm{f}}$ in $w_{k}^{(2)}$. In particular, it vanishes identically when $n_{\mathrm{f}}=0$. It can thus be neglected for small $n_{\mathrm{f}}$. On the other hand, for larger $n_{\mathrm{f}}$, but not near point $\mathrm{T}$ which would require a refined treatment, the wide separation between the two transition lines guarantees that the colleative dynamics will be fast and regular at the diffusiqn localization transition. It results that the singular ta ged-particle dynamics is cut off in the bilinear term, which generates a fast and smoothly varying contribution to $m^{\mathrm{MSD}}(t)$. Thus it is in general enough to set

$$
m^{\mathrm{MSD}}(t)=\gamma \delta(t)+\int_{0}^{+\infty} \frac{k^{4} d k}{6 \pi^{2}} \hat{h}_{k}^{\mathrm{ss}(\mathrm{b})} \phi_{k}^{\mathrm{s}}(t)
$$

with an appropriate renormalization of $\gamma$. Then, combining Eqs. (C1) with $\tilde{D}_{q}(z) \simeq \tilde{D}(z),(\mathrm{C} 3)$, and the Laplace transform of Eq. (C4), a transcendental equation for $\tilde{D}(z)$ is obtained, which reads

$$
\left(\frac{m_{\mathrm{s}}}{k_{B} T} z+i \gamma\right) \tilde{D}(z)=-1+\int_{0}^{+\infty} \frac{k^{4} d k}{6 \pi^{2}} \frac{\hat{h}_{k}^{\mathrm{ss}(\mathrm{b})}}{k^{2}+z / \tilde{D}(z)} .
$$

From the solution of this equation, which is the core result of the proposed generalized hydrodynamic approximation, all relevant dynamical quantities can be computed.

The diffusive and localized phases are characterized by different small $z$ behaviors of $\tilde{D}(z)$. In the diffusive phase,

$$
\lim _{z \rightarrow i 0} \tilde{D}(z)=i D,
$$

where $D$ is the finite long-time diffusion coefficient. From Eq. (C5), one finds

$$
D=\frac{1}{\gamma}\left[1-\frac{1}{3} h^{\mathrm{ss}(\mathrm{b})}(r=0)\right] .
$$

In the localized phase,

$$
\tilde{D}(z) \sim z r_{1}^{2},
$$


where $r_{1}$ is the finite localization length, which obeys

$$
\int_{0}^{+\infty} \frac{k^{4} d k}{6 \pi^{2}} \frac{\hat{h}_{k}^{\mathrm{ss}(\mathrm{b})}}{k^{2}+r_{1}^{-2}}=1 .
$$

Using a large $r_{1}$ expansion of Eq. (C9), one easily shows that the divergence of $r_{1}$ and the vanishing of $D$ both occur when

$$
h^{\mathrm{ss}(\mathrm{b})}(r=0)=3 .
$$

So, as anticipated, the present theory indeed describes a bona fide diffusion-localization transition scenario, with the above condition providing an implicit equation of the transition line.
[1] Proceedings of the International Workshop on Dynamics in Confinement, edited by R. Zorn, B. Frick, and H. Büttner [J. Phys. IV 10, Pr7-1 (2000)].

[2] Proceedings of the Second International Workshop on Dynamics in Confinement, edited by B. Frick, M. Koza, and R. Zorn [Eur. Phys. J. E 12, 3 (2003)].

[3] Proceedings of the Third International Workshop on Dynamics in Confinement, edited by M. Koza, B. Frick, and R. Zorn [Eur. Phys. J. Special Topics 141, IV (2007)].

[4] S. Havlin and D. Ben-Avraham, Adv. Phys. 51, 187 (2002).

[5] J.-P. Bouchaud and A. Georges, Phys. Rep. 195, 127 (1990).

[6] R. Kimmich, Chem. Phys. 284, 253 (2002).

[7] M. Alcoutlabi and G. B. McKenna, J. Phys.: Condens. Matter 17, R461 (2005).

[8] C. Alba-Simionesco, B. Coasne, G. Dosseh, G. Dudziak, K. E. Gubbins, R. Radhakrishnan, and M. SliwinskaBartkowiak, J. Phys.: Condens. Matter 18, R15 (2006).

[9] H. Sillescu, J. Non-Cryst. Solids 243, 81 (1999).

[10] M. D. Ediger, Ann. Rev. Phys. Chem. 51, 99 (2000).

[11] R. Richert, J. Phys.: Condens. Matter 14, R703 (2002).

[12] U. Bengtzelius, W. Götze, and A. Sjölander, J. Phys. C 17, 5915 (1984).

[13] E. Leutheusser, Phys. Rev. A 29, 2765 (1984).

[14] W. Götze, in Liquids, Freezing and Glass Transition, edited by J.-P. Hansen, D. Levesque, and J. Zinn-Justin (North-Holland, Amsterdam, 1991), pp. 287-503.

[15] W. Götze and L. Sjögren, Rep. Prog. Phys. 55, 241 (1992).

[16] W. Götze, J. Phys.: Condens. Matter 11, A1 (1999).

[17] V. Krakoviack, Phys. Rev. Lett. 94, 065703 (2005).

[18] V. Krakoviack, J. Phys.: Condens. Matter 17, S3565 (2005).

[19] V. Krakoviack, Phys. Rev. E 75, 031503 (2007).

[20] W. G. Madden and E. D. Glandt, J. Stat. Phys. 51, 537 (1988).

[21] W. G. Madden, J. Chem. Phys. 96, 5422 (1992).

[22] J. Bosse and J. S. Thakur, Phys. Rev. Lett. 59, 998 (1987).

[23] J. S. Thakur and J. Bosse, Phys. Rev. A 43, 4378 (1991).

[24] J. S. Thakur and J. Bosse, Phys. Rev. A 43, 4388 (1991).

[25] W. Götze, E. Leutheusser, and S. Yip, Phys. Rev. A 23, 2634 (1981).

[26] E. Leutheusser, Phys. Rev. A 28, 2510 (1983).

[27] G. Szamel, Europhys. Lett. 65, 498 (2004).

[28] J. A. Given and G. Stell, J. Chem. Phys. 97, 4573 (1992).

[29] E. Lomba, J. A. Given, G. Stell, J. J. Weis, and D.
Levesque, Phys. Rev. E 48, 233 (1993).

[30] J. A. Given and G. Stell, Physica A 209, 495 (1994).

[31] M.-L. Rosinberg, G. Tarjus, and G. Stell, J. Chem. Phys. 100, 5172 (1994).

[32] E. Paschinger and G. Kahl, Phys. Rev. E 61, 5330 (2000).

[33] M. A. Chávez-Rojo, R. Juárez-Maldonado, and M. Medina-Noyola, Phys. Rev. E 77, 040401(R) (2008).

[34] In fact, by an appropriate use of the replica trick or of the cluster diagram expansion technique and a suitable redefinition of the blocked direct correlation functions, one can demonstrate the universality of the OZ equations reported in Refs. [35, 36], which are actually applicable in any situation involving a fluid in a statistically homogeneous random environment, be it based on randomly placed particles or not (V. Krakoviack, in preparation).

[35] G. I. Menon and C. Dasgupta, Phys. Rev. Lett. 73, 1023 (1994).

[36] E. Kierlik, M.-L. Rosinberg, and G. Tarjus, J. Stat. Phys. 94, 805 (1999).

[37] W. Götze, Amorphous and Liquid Materials, Vol. 118 of NATO Advanced Study Institute, Series E: Applied Sciences (Nijhoff, Dordrecht, 1987), pp. 34-81.

[38] A. Meroni, D. Levesque, and J.-J. Weis, J. Chem. Phys. 105, 1101 (1996).

[39] In Ref. [19], it was found that, in order to have coincidence between the MCT equations for the residual dynamics of a bulk system in its ideal glassy phase and those for the collective dynamics in a QA binary mixture, one has both to neglect the blocked direct correlation functions and to replace one of the Fourier transformed direct correlation functions appearing in the former by a constant term equal to $1 / n_{f}$.

[40] T. Franosch, M. Fuchs, W. Götze, M. R. Mayr, and A.P. Singh, Phys. Rev. E 55, 7153 (1997).

[41] M. Fuchs, W. Götze, and M. R. Mayr, Phys. Rev. E 58, 3384 (1998).

[42] W. Götze, Philos. Mag. B 43, 219 (1981).

[43] E. Leutheusser, Phys. Rev. A 28, 1762 (1983).

[44] F. Höfling, E. Frey, and T. Franosch, unpublished.

[45] L. Sjögren, Phys. Rev. A 33, 1254 (1986).

[46] W. Götze and R. Haussmann, Z. Phys. B: Condens. Matter 72, 403 (1988).

[47] T. Franosch and W. Götze, J. Phys.: Condens. Matter 6, 4807 (1994).

[48] F. Höfling, T. Franosch, and E. Frey, Phys. Rev. Lett. 96, 165901 (2006).

[49] F. Höfling and T. Franosch, Phys. Rev. Lett. 98, 140601 (2007). 
[50] F. Höfling, T. Munk, E. Frey, and T. Franosch, J. Chem. Phys. 128, 164517 (2008).

[51] B. J. Sung and A. Yethiraj, Phys. Rev. Lett. 96, 228103 (2006).

[52] B. J. Sung and A. Yethiraj, J. Phys. Chem. B 112, 143 (2008).

[53] B. J. Sung and A. Yethiraj, J. Chem. Phys. 128, 054702 (2008).

[54] S. Babu, J. C. Gimel, and T. Nicolai, J. Phys. Chem. B 112, 743 (2008).

[55] G. Foffi, K. A. Dawson, S. V. Buldyrev, F. Sciortino, E. Zaccarelli, and P. Tartaglia, Phys. Rev. E 65, 050802(R) (2002).

[56] E. Zaccarelli, G. Foffi, K. A. Dawson, S. V. Buldyrev, F. Sciortino, and P. Tartaglia, Phys. Rev. E 66, 041402 (2002).

[57] G. Foffi, F. Sciortino, P. Tartaglia, E. Zaccarelli, F. Lo Verso, L. Reatto, K. A. Dawson, and C. N. Likos, Phys. Rev. Lett. 90, 238301 (2003).

[58] E. Zaccarelli, H. Löwen, P. P. F. Wessels, F. Sciortino, P. Tartaglia, and C. N. Likos, Phys. Rev. Lett. 92, 225703 (2004).

[59] C. Mayer, E. Zaccarelli, E. Stiakakis, C. N. Likos, F. Sciortino, A. Munam, M. Gauthier, N. Hadjichristidis, H. Iatrou, P. Tartaglia, H. Löwen, and D. Vlassopoulos, Nature Mater. 7, 780 (2008).

[60] S.-H. Chong, A. J. Moreno, F. Sciortino, and W. Kob, Phys. Rev. Lett. 94, 215701 (2005).

[61] A. J. Moreno, S.-H. Chong, W. Kob, and F. Sciortino, J. Chem. Phys. 123, 204505 (2005).

[62] C. Oleksy, J. Phys. A 24, L751 (1991).

[63] In the case of Newtonian dynamics, the same equation holds with $D_{0}$ replaced by $\gamma^{-1}, \gamma$ being the instantaneous part of the memory kernel $m^{\mathrm{MSD}}(t)$.

[64] W. Götze and M. Sperl, Phys. Rev. E 66, 011405 (2002).

[65] M. Sperl, Phys. Rev. E 68, 031405 (2003).

[66] M. Sperl, Phys. Rev. E 69, 011401 (2004).

[67] W. Götze and M. Sperl, J. Phys.: Condens. Matter 16, S4807 (2004).

[68] G. H. Vineyard, Phys. Rev. 110, 999 (1958).

[69] J. P. Boon and S. Yip, Molecular hydrodynamics (Dover, New York, 1991).

[70] Examples abound. For instance, it is well known that, in the bulk, the total and single-particle density correlation functions do not have the same hydrodynamic behavior and that there is no simple relation between them in this regime [69]. A few simple toy models also provide interesting illustrations of this decoupling phenomenon. In the lattice gas model with hard particles hopping at a constant rate between nearest-neighbor sites, it has been shown that the normalized connected density correlation function is density-independent and exponential [W. Dieterich, P. Fulde, and I. Peschel, Adv. Phys. 29, 527 (1980); R. Kutner, Phys. Lett. A 81, 239 (1981)], while the tagged-particle motion is density-dependent and nontrivial $[\mathrm{K}$. W. Kehr, R. Kutner, and K. Binder, Phys. Rev. B 23, 4931 (1981)]. In the Jepsen gas, the dynamics of the collective density fluctuations is densityindependent and ballistic, while that of the single-particle density fluctuations is density-dependent and diffusive at long times [D. W. Jepsen, J. Math. Phys. 6, 405 (1965)].

[71] M. Arndt, R. Stannarius, W. Gorbatschow, and F. Kremer, Phys. Rev. E 54, 5377 (1996).

[72] M. Arndt, R. Stannarius, H. Groothues, E. Hempel, and F. Kremer, Phys. Rev. Lett. 79, 2077 (1997).

[73] G. Viramontes-Gamboa, J. L. Arauz-Lara, and M. Medina-Noyola, Phys. Rev. Lett. 75, 759 (1995)

[74] G. Viramontes-Gamboa, M. Medina-Noyola, and J. L. Arauz-Lara, Phys. Rev. E 52, 4035 (1995).

[75] P. Gallo, R. Pellarin, and M. Rovere, Europhys. Lett. 57, 212 (2002)

[76] P. Gallo, R. Pellarin, and M. Rovere, Phys. Rev. E 67, 041202 (2003).

[77] P. Gallo, R. Pellarin, and M. Rovere, Phys. Rev. E 68, 061209 (2003).

[78] A. Attili, P. Gallo, and M. Rovere, J. Chem. Phys. 123, 174510 (2005).

[79] K. Kim, Europhys. Lett. 61, 790 (2003).

[80] R. Chang, K. Jagannathan, and A. Yethiraj, Phys. Rev. E 69, 051101 (2004).

[81] J. Mittal, J. R. Errington, and T. M. Truskett, Phys. Rev. E 74, 040102(R) (2006).

[82] M. Nauroth and W. Kob, Phys. Rev. E 55, 657 (1997).

[83] W. Kob, M. Nauroth, and F. Sciortino, J. Non-Cryst. Solids 307-310, 181 (2002). 